\title{
NOUVELLES DONNÉES SUR LA LIGNÉE DIPETALONEMA
}

\author{
(Filarioidea, Nematoda).
}

\author{
O. BAIN, M. BAKER et A. G. CHABAUD*
}

RÉSUMÉ. La " lignée Dipetalonema " paraît pouvoir être scindée en 4 groupes : I : les espèces australiennes; II : les espèces paléoendémiques d'Amérique du Sud ; III : les espèces rattachables au groupe Tetrapetalonema; IV : les espèces rattachables au groupe Acanthocheilonema. Plusieurs espèces du $4^{\mathrm{e}}$ groupe font l'objet de ce travail, ainsi que le genre Loxodontofilaria, insuffisamment connu pour être classé.

Description de Loxodontofilaria asiatica n. sp., parasite d'Elephas indicus en Birmanie, Cercopithifilaria degraaffi n. sp., parasite de Papio ursinus en Afrique du Sud, C. cephalophi n. sp., parasite de Cephalophus dorsalis et $C$. gabonensis n. sp., parasite d'Atherurus africanus au Gabon.

Compléments morphologiques sur Cercopithifilaria didelphis, C. rugosicauda, Acanthocheilonema pachycephalum, A. viteae, Molinema dessetae, Dipetalonema gracile, Orihelia sp., Skrjabinofilaria skrjabini, Breinlia (B.) spratti, Litomosa sp., Loxodontofilaria hippopotami.

Création de Yatesia n. gen., espèce-type Yatesia hydrochoerus (Yates, 1980), en raison de caractères spécialisés de l'extrémité postérieure du corps. Le genre Cercopithifilaria est utilisé pour regrouper les espèces considérées comme des Acanthocheilonema spécialisés. Chenofilaria est mis en synonymie d'Acanthocheilonema. Loxodontofilaria groupe les 3 espèces de Filaires des Éléphants, L. loxodontis, L. gossi, L. asiatica n. sp., et celle de l'Hippopotame. L. hippopotami. D. okapiae est considéré comme species inquirenda.

L’interprétation donnée pour la faune néotropicale est la suivante :

- Skrjabinofilaria, Orihelia, Dasypafilaria et Dipetalonema seraient des paléoendémiques véritables.

- Molinema et Ackertia, d'une part, Yatesia, d'autre part, correspondraient à des formes d'origine africaine introduites à la fin de l'Éocène, lors du passage des Rongeurs de l'Afrique vers l'Amérique. La capture par les Reptiles américains (genre Macdonaldius) pourrait s'être faite à cette époque.

- De façon inattendue, les 2 espèces parasites des Didelphis seraient des captures tardives d'origine néarctique, $A$. pricei à partir d'Acanthocheilonema de Carnivores et $C$. didelphis à partir d'un Cercopithifilaria de Mammifère Euthérien.

\section{New data on the evolutionary line of Dipetalonema (Filarioidea, Nematoda)}

SUMMARY. The evolutionary line of Dipetalonema can apparently be divided into four groups: I: Australian species; II: paleoendemic South American species; III: the Tetrapetalonema group; IV: the Acanthocheilonema group. Loxodontofilaria at present insufficiently known to be classified and several species belonging to the Acanthocheilonema group are the object of the present study.

* Laboratoire de Zoologie-Vers, associé au CNRS, Muséum National d'Histoire Naturelle, 61, rue Buffon, F 75231 Paris Cedex 05.

Accepté le 29 août 1982 . 
Descriptions are given of Loxodontofilaria asiatica n. sp., parasite of Elephas indicus in Burma, Cercopithifilaria degraaffi n. sp., parasites of Papio ursinus in South Africa, C. cephalophi n. sp., parasite of Cephalophus dorsalis and C. gabonensis n. sp., parasite of Atherurus africanus in Gabon. Additional morphological data are given on Cercopithifilaria didelphis, C. rugosicauda, Acanthocheilonema pachycephalum, A. viteae, Molinema dessetae, Dipetalonema gracile, Orihelia sp., Skrjabinofilaria skrjabini, Breinlia (B.) spratti, Litomosa sp., Loxodontofilaria hippopotami.

Yatesia n. gen. with type species Yatesia hydrochoerus (Yates, 1980), is proposed, distinguished by specialized characters of the posterior extremity. The genus Cercopithifilaria is used to accomodate species considered as specialized Acanthocheilonema. Chenofilaria is placed in synonymy with Acanthocheilonema. Loxodontofilaria includes the three filarid species from elephants, L. loxodontis, L. gossi, L. asiatica n. sp. and the species from the Hippopotamus, L. hippopotami; D. okapiae is considered a species inquirenda.

The interpretation given for the neotropical fauna is the following:

- Skrjabinofilaria, Orihelia, Dasypafilaria and Dipetalonema may be true paleoendemics in South America.

- Molinema and Ackertia on the one hand and Yatesia on the other may be forms of African origin introduced at the end of the Eocene during the migration of African rodents into South America. The capture in American reptiles (the genus Macdonaldius) could have occurred during this period.

- Surprisingly, the two species of Dipetalonema in Didelphis may be late captures of neartic origin: A. pricei from Acanthocheilonema in carnivores and $C$. didelphis from a Cercopithifilaria in eutherian mammals.

\section{Introduction}

De nombreuses données nouvelles ont été accumulées au cours des six dernières années sur les Filaires de la lignée Dipetalonema.

L'objet de cette note est de contribuer à l'étude morphologique de ce groupe et de voir dans quelle mesure les données nouvelles modifient les hypothèses formulées sur son évolution.

Il apparaîtra que les niveaux génériques ou subgénériques qui étaient attribués aux différents taxas ne correspondent plus à la conception d'ensemble. La plupart des sous-genres ont donc été élevés au niveau du genre.

\section{I - Loxodontofilaria asiatica $\mathrm{n} . \mathrm{sp}$.}

\section{Matériel}

1 우 avec microfilaires (holotype), 1 우 sans microfilaire (paratype), Onderstepoort. Coll. no 0.356 .

Hôte : Elephas indicus L., en Birmanie ; localisation chez l'hôte inconnue.

\section{Morphologie (fig. 1).}

Corps avec un seul renflement, à la hauteur de la vulve.

Papilles de la tête disposées suivant la figure $1 \mathrm{~B}$ et $C$; papilles labiales externes latérales vestigiales ; les 4 autres papilles labiales externes presque disposées en carré, 
les 4 papilles céphaliques en rectangle étiré latéralement ; amphides au même niveau que les papilles labiales externes. Bouche et cavité buccale un peu allongées dorsoventralement. Cavité buccale entourée par la cuticule céphalique épaissie; pas d'anneau cuticulaire préœsophagien.

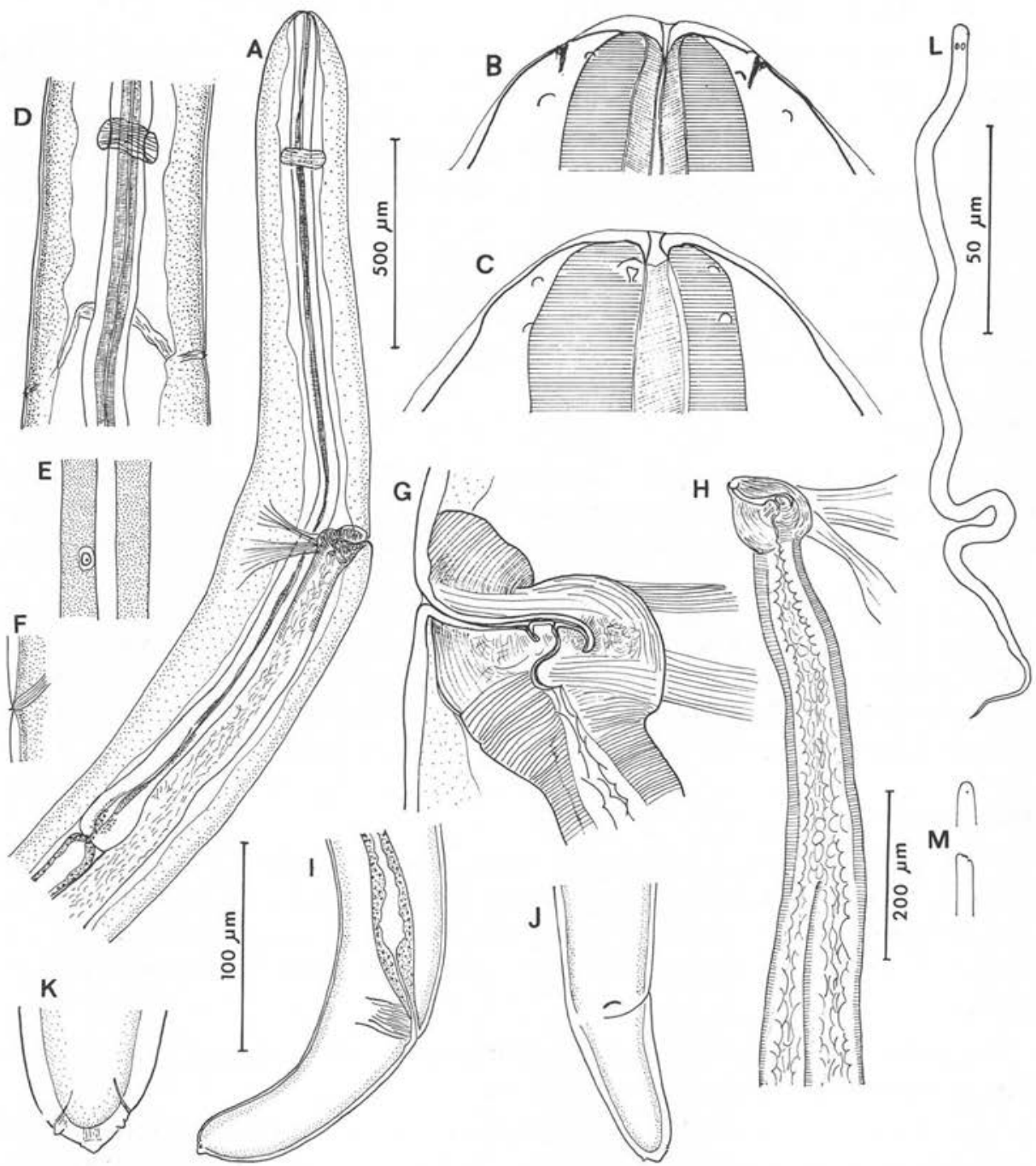

FIG. I. - Loxodontofilaria asiatica n. sp., 우; A : région antérieure, vue latérale ; B et C : tête, vues médiane et latérale ; $\mathrm{D}$ : position des deirides, vue médiane ; E et F : une deiride, vue de face et de profil ; $\mathrm{G}$ : vagin ; $\mathrm{H}$ : ovéjecteur ; I et $\mathrm{J}$ : région caudale, vues latérale et ventrale ; $\mathrm{K}$ : extrémité caudale, vue ventrale; $\mathrm{L}$ : microfilaire utérine ; $\mathrm{M}$ : idem, extrémité céphalique : en haut crochet vu de face, en bas, vu de profil (A, éch. $500 \mu \mathrm{m} ; \mathrm{D}, \mathrm{H}, \mathrm{I}, \mathrm{J}$ éch. $200 \mu \mathrm{m} ; \mathrm{E}, \mathrm{F}, \mathrm{G}$, K éch. roo $\mu \mathrm{m}$; B, C, L, M éch. $50 \mu \mathrm{m})$. 
Esophage long, non divisé, terminé ou non par un renflement bulbeux ; lumière œsophagienne bien cuticularisée en forme d' " $\mathrm{y}$ " en section transversale ; granules de sécérétions irrégulièrement dispersés (dans le renflement terminal chez la † paratype).

Deirides présentes.

Vulve à mi-hauteur de l'œsophage. Portion impaire de l'ovéjecteur courte ; vagin musculeux constitué par 1 segment proximal perpendiculaire à la paroi du corps et tapissé de cuticule (vagina vera), et par 1 segment distal, non cuticularisé, inséré sur le côté du tube précédent et formant avec lui un angle droit ; muscles suspendant le vagin au corps très marqués. Opisthodelphie. Vésicule séminale très longue (presque $1 \mathrm{~cm}$ ).

Queue légèrement courbée vers la face dorsale; extrémité caudale conique ; 2 petits tubercules subterminaux latéraux et phasmides près de ces tubercules.

Microfilaires sans gaine ni corps interne ; extrémité céphalique obtuse ou biseautée suivant l'orientation; queue longue et effilée.

\section{Dimensions}

ㅇ holotype : corps long de $180 \mathrm{~mm}$ et large de $295 \mu \mathrm{m}$; anneau nerveux et deirides à 380 et $700 \mu \mathrm{m}$ de l'apex; œsophage long de $2600 \mu \mathrm{m}$; vulve à $1300 \mu \mathrm{m}$ de l'apex ; queue longue de $320 \mu \mathrm{m}$; microfilaires longues de $248-252 \mu \mathrm{m}$ sur $5,5 \mu \mathrm{m}$ de large.

ㅇ paratype : corps long de $190 \mathrm{~mm}$ et large de $420 \mu \mathrm{m}$; anneau nerveux et deirides à $440 \mu \mathrm{m}, 760$ et $820 \mu \mathrm{m}$ de l'apex; œsophage long de $2740 \mu \mathrm{m}$ et épais de $50 \mu \mathrm{m}$; vulve à $1550 \mu \mathrm{m}$ de l'apex ; portion impaire de l'ovéjecteur longue de $630 \mu \mathrm{m}$, sphincter épais de 140 sur $110 \mu \mathrm{m}$, ovaires à 12,25 et 19,85 mm de l'extrémité caudale, vésicule séminale longue de $7500 \mu \mathrm{m}$; queue longue de $350 \mu \mathrm{m}$.

\section{Discussion}

En dehors de la Filaire décrite ici, on ne connaît, semble-t-il, chez l'Éléphant d'Asie qu'une microfilaire sanguine, signalée par Evans et Rennie, en Birmanie, en 1910 (in Baylis, 1939) ; elle est beaucoup plus courte que nos microfilaires (180 $\mu \mathrm{m}$ au lieu de 248-252 $\mu \mathrm{m}$ ), et pourrait être un embryon sanguicole d'Habronème (Khalil, 1929).

La Filaire d'Elephas indicus est très proche des 2 espèces connues chez Loxodonta africana, en Afrique, mais elle en est distincte :

- Loxodontofilaria loxodontis Berghe et Gillain, 1939, en Afrique équatoriale, parasite sous-cutané, a un œsophage deux fois plus court, une vulve deux fois plus antérieure et une microfilaire plus courte $(200 \mu \mathrm{m})$.

- L. gossi (Baylis, 1923), en Afrique orientale et australe, parasite des veines portes du foie (Basson et coll., 1971), a un œsophage plus court, une vulve nettement plus postérieure et une microfilaire plus longue $(275 \mu \mathrm{m})$. 
Les spécimens de l'Éléphant d'Asie représentent donc une espèce nouvelle, Loxodontofilaria asiatica $\mathrm{n}$. sp.

Il avait été envisagé (Chabaud et Bain, 1976) que les Filaires d'Éléphants pourraient constituer la base d'une petite lignée évoluée de Dipetalonema (sous-genre Loxodontofilaria), propre aux Ongulés d'Afrique : Éléphants, Hippopotame, Okapi et Bovidés).

Nous connaissons bien maintenant plusieurs espèces parasites des Bovidés africains : ruandae Fain et Hérin, 1955, dermicola Fain, 1977, faini Chabaud et coll., 1978 et cephalophi n. sp. (cf. plus loin).

Elles constituent un groupe homogène qui ne se sépare pas nettement de Cercopithifilaria Eberhard, 1980 (cf. plus loin) mais dont les femelles s'éloignent de celles qui sont parasites des Éléphants : anneau préœsophagien présent, œsophage grêle, déirides absentes.

Loxodonfilaria paraît donc devoir être restreint actuellement aux trois Filaires parasites d'Éléphants, dont les ô sont inconnus et peut être à $L$. hippopotami (Leiper, 1910) (cf. description suivante) au ơ également inconnu, et que rien ne sépare de ce que l'on sait sur Loxodontofilaria : anneau préœsophagien absent, œsophage non grèle, deirides présentes.

La Filaire de l'Okapi, D. okapiae Fain, 1948, dont nous avons pu observer le matériel type*, malheureusement très endommagé (il ne reste plus que la région antérieure du ô et la région postérieure de la of) est species inquirenda; elle présente quelques caractères communs avec les Dipetalonema de Bovidés (petite taille, œsophage grèle), mais il n'y a aucune trace de capsule buccale comme chez L. asiatica n. sp.

\section{II — Loxodontofilaria hippopotami \\ (Leiper, 1910)}

\section{Matériel}

1 우 avec microfilaires, 3 extrémités antérieures, 1 extrémité postérieure et quelques autres fragments de 우; Onderstepoort. Coll. $\mathrm{n}^{\circ} 1326$ (=214 KB) ; 1 우 sans microfilaires, Onderstepoort. Coll. no 1360 (=215 KB).

Hôte: Hippopotamus amphibius L. en Afrique du Sud (Krüger National Park) ; parasites dans la cavité générale.

\section{Morphologie (fig. 2).}

Corps avec un léger renflement circulaire un peu en arrière de l'anneau nerveux ; en avant du renflement, région céphalique fortement amincie.

Papilles de la tête disposées suivant les figures $C, E, F$; papilles labiales externes latérales vestigiales, près des amphides.

* Nous remercions M. Fain et M. Puylaert qui nous ont très aimablement prêté ce matériel. 

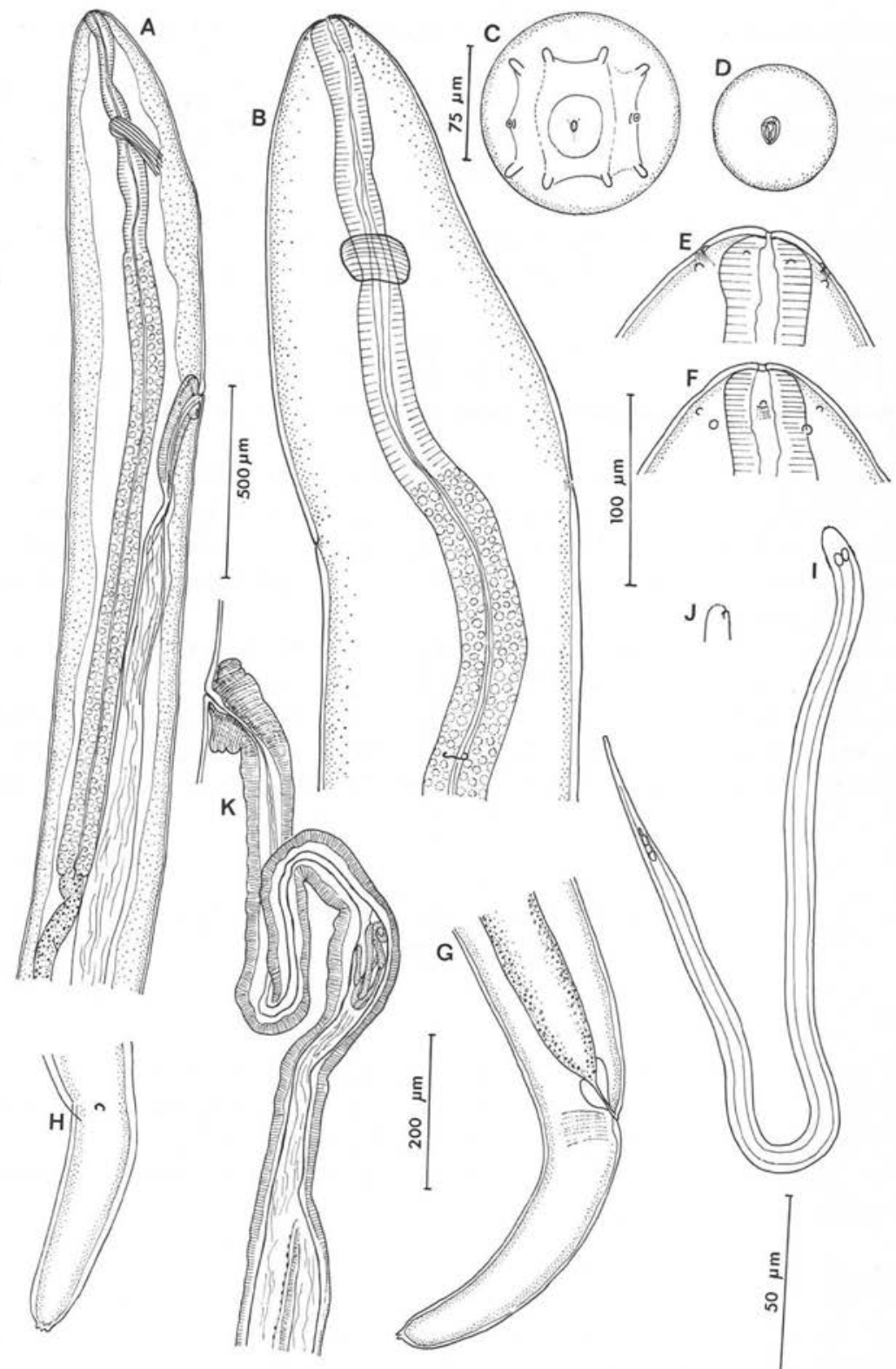

ㅇ
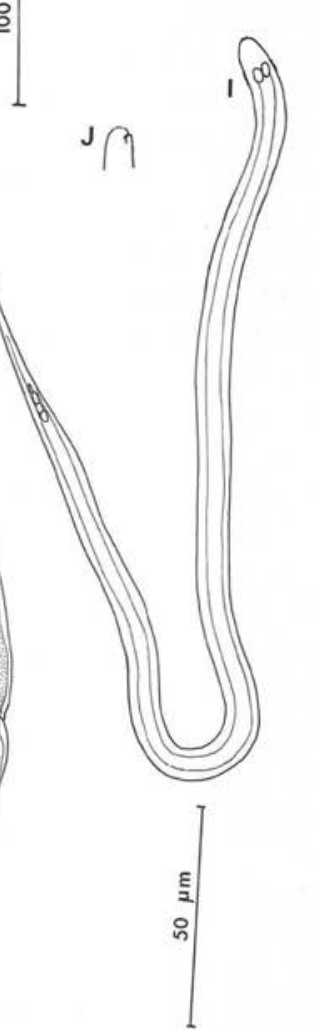

FIG. 2. - Loxodontofilaria hippopotami, 우 A : région antérieure, vue latérale; B : idem, position des deirides, vue médiane ; C : tête, vue apicale; D : bouche et apex de l'œsophage, vue apicale ; $\mathrm{E}$ et $\mathrm{F}$ : tête, vues médiane et latérale; $\mathrm{G}$ et $\mathrm{H}$ : région caudale, vues latérale et médiane ; I : microfilaire utérine ; J : idem, tête, crochet de profil ; $\mathrm{K}$ : ovéjecteur (A, éch. $500 \mu \mathrm{m} ; \mathrm{B}, \mathrm{G}, \mathrm{H}, \mathrm{K}$ éch. $200 \mu \mathrm{m}$; E, F éch. $100 \mu \mathrm{m}$; C, éch. $75 \mu \mathrm{m} ; \mathrm{D}, \mathrm{I}, \mathrm{J}$ éch. $50 \mu \mathrm{m})$. 
Bouche petite, en ovale légèrement étiré dans le plan médian. Cavité buccale très réduite également ovale en section transversale et entourée par la cuticule céphalique légèrement épaissie ; pas d'anneau cuticulaire préœsophagien.

Esophage divisé ; portion glandulaire un peu plus épaisse que la portion musculaire ; lumière œsophagienne à section transversale en " $\mathrm{y}$ » et bien cuticularisée.

Deirides présentes.

Vulve à mi-hauteur de l'œsophage. Ovéjecteur simple (pas de sphincter en S) rapidement divisé en 2 branches. Opisthodelphie.

Queue courbée vers la face dorsale ; extrémité caudale terminée par trois pointes coniques de tailles presque égales, une médiane pointue et deux latérales émoussées.

Microfilaire grande, sans gaine ni corps interne, à cuticule épaisse et fortement striée ; filament caudal anucléé assez épais.

\section{Dimensions}

ㅇ avec microfilaires, lot $214 \mathrm{~KB}$ : corps long de $95 \mathrm{~mm}$ et large de $480 \mu \mathrm{m}$; anneau nerveux et vulve à 380 et $1300 \mu \mathrm{m}$ de l'apex; œsophage long de $2550 \mu \mathrm{m}$ (portion musculaire longue de $700 \mu \mathrm{m}$ ) ; ovéjecteur long de $1600 \mu \mathrm{m}$; queue longue de $480 \mu \mathrm{m}$.

ㅇ sans microfilaires, lot $215 \mathrm{~KB}$ : corps long de $90 \mathrm{~mm}$ et large de $375 \mu \mathrm{m}$; anneau nerveux, deirides et vulve à $380 \mu \mathrm{m}, 860 \mu \mathrm{m}$ et $1300 \mu \mathrm{m}$ de l'apex ; cavité buccale haute de $6 \mu \mathrm{m}$; œsophage long de $2800 \mu \mathrm{m}$ (portion musculaire longue de $800 \mu \mathrm{m}$ ) ; queue longue de $530 \mu \mathrm{m}$; pointes caudales longues de $8 \mu \mathrm{m}$.

Microfilaires : corps long de $355,380,360$ et $370 \mu \mathrm{m}$ et large de 9-10 $\mu \mathrm{m}$.

\section{Discussion}

L'unique Filaire connue jusqu'à présent chez l'Hippopotame, Loxodontofilaria hippopotami (Leiper, 1910), est décrite d'Ouganda et récoltée dans le tissu souscutanée. La microfilaire n'est pas connue et le seul caractère qui diffère entre cette espèce et nos spécimens - pointe caudale axiale émoussée ou pointue — nous paraît insuffisant pour distinguer notre matériel.

Les affinités de la Filaire d'Hippopotame ne peuvent être établies puisque le $\widehat{\sigma}$ est inconnu mais son maintien dans le genre Loxodontofilaria se justifie par la présence de caractères communs avec les Filaires d'Éléphants (pas d'anneau cuticulaire préœsophagien, deirides présentes, œsophage développé).

\section{III - Acanthocheilonema pachycephalum (Ortlepp, 1961)}

\section{Matériel}

2 ㅇ, 8 extrémités antérieures et 7 extrémités postérieures de + et $2 \hat{0}$, Ondersteeport. Coll. no 2184 (=216 KB).

Hôte : Poecilogale albinucha (Gray), Mustelidé capturé en Afrique du Sud $\left(33^{\circ} 15^{\prime} 11^{\prime \prime} \mathrm{S}\right.$ et $\left.27^{\circ} 5^{\prime} 0^{\prime \prime} \mathrm{E}\right)$; localisation chez l'hôte non précisée. 


\section{Morphologie (fig. 3 et 4)}

Pas de renflements sur le corps. Région antérieure avec un brusque rétrécissement subapical délimitant une petite calotte céphalique. Papilles de la tête représentées sur les figures $3 \mathrm{H}$ à $J$ et $4 \mathrm{D}$ et $\mathrm{E}$ : papilles céphaliques disposées suivant un rectangle légèrement étiré latéralement, papilles labiales externes suivant un carré ou un rectangle légèrement étiré dans le plan médian ; amphides presque à la hauteur des papilles labiales externes; papilles labiales latérales externes non observées.

Bouche ronde ; cavité buccale plus haute que large, à section transversale arrondie, délimitée par une capsule buccale formée de deux segments, un antérieur allongé, un postérieur très court.

Esophage divisé avec portion glandulaire deux fois plus épaisse que la portion musculaire ; deirides présentes.

Femelle (fig. 3) : vulve vers le tiers postérieur de l'œsophage glandulaire ; ovéjecteur impair long et vagin musculeux bien développé comprenant - un segment proximal coudé tapissé de cuticule et à lumière aplatie - une chambre - un segment distal à lumière très étroite. Opisthodelphie.

Queue mince, légèrement courbée vers la face dorsale ; extrémité caudale arrondie ornée par trois courtes pointes, une terminale et deux latérales ventrales près desquelles débouchent les phasmides.

Mâle (fig. 4): Area rugosa représentée sur la figure 4 F; spicule droit en 2 parties: manche antérieur et longue gouttière postérieure qui porte sur la face dorsale des indentations subterminales. Spicule gauche à lame ornée d'une vésicule membraneuse dans le $1 / 3$ antérieur et à extrémité distale pointue. Papilles caudales disposées suivant la figure $4 \mathrm{G}$ à $J$. Extrémité caudale ornée par 3 ou 4 tubercules subterminaux et ventraux; phasmides à la base des tubercules les plus latéraux.

Microfilaire : pas de gaine ni de corps interne. Crochet céphalique long ; forme de la tête variable selon l'orientation : petite calotte céphalique brusquement plus étroite que le corps quand le crochet est vu de face, encoche transversale visible sous le crochet quand celui-ci est de profil (fig. 3, $O, N$ ) ; région caudale effilée avec filament anucléé très fin.

\section{Dimensions}

우 : corps long de $52 \mathrm{~mm}$, large de $360 \mu \mathrm{m}$; anneau nerveux et deirides à 240 et $380-400 \mu \mathrm{m}$ de l'extrémité céphalique ; capsule buccale haute de $13 \mu \mathrm{m}$; œsophage long de $2000 \mu \mathrm{m}$ et portion musculaire longue de $375 \mu \mathrm{m}$; vulve à $1360 \mu \mathrm{m}$ de l'extrémité antérieure ; ovéjecteur impair long de $2400 \mu \mathrm{m}$; queue longue de $375 \mu \mathrm{m}$ et pointes caudales hautes de $3 \mu \mathrm{m}$.

Autre $ᄋ$ : corps long de $43 \mathrm{~mm}$ et large de $280 \mu \mathrm{m}$; œsophage long de $1500 \mu \mathrm{m}$; vulve à $1270 \mu \mathrm{m}$ de l'extrémité antérieure; queue longue de $430 \mu \mathrm{m}$ et pointes caudales hautes de 3,5-4 $\mu \mathrm{m}$.

Un $\widehat{o}$ : corps long de $22 \mathrm{~mm}$ et large de $180 \mu \mathrm{m}$; anneau nerveux, pore excréteur et deirides à $220 \mu \mathrm{m}, 350 \mu \mathrm{m}$ et $450-470 \mu \mathrm{m}$ de l'extrémité antérieure ; œsophage long 


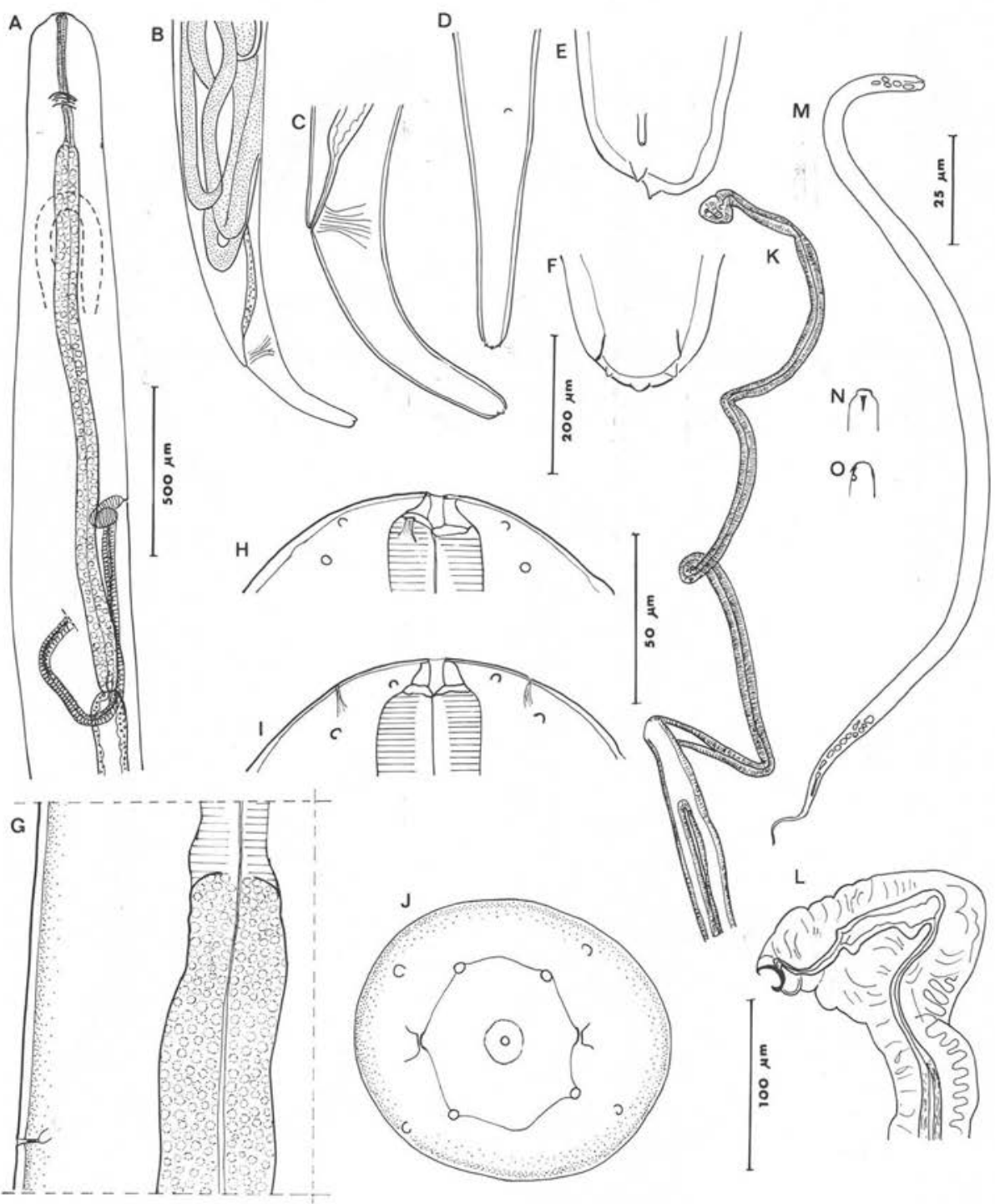

FIG. 3. - Acanthocheilonema pachycephalum, 우; A : région antérieure, vue latérale (en pointillés la boucle utérine la plus antérieure) ; B, C, D : région caudale en vues latérales et vue médiane ; $\mathrm{E}$ et $\mathrm{F}$ : extrémité caudale, vues latérale et ventrale; $\mathrm{G}$ : position d'une deiride, vue médiane ; $\mathrm{H}$, I et $\mathrm{J}$ : tête, vues latérale, médiane et apicale ; $\mathrm{K}$ : ovéjecteur ; $\mathrm{L}$ : vagin ; $\mathrm{M}$ : microfilaire utérine; $\mathrm{N}$ et $\mathrm{O}$ : idem, tête, crochet vu de face ou de profil (A, B, K éch. $500 \mu \mathrm{m} ; \mathrm{C}, \mathrm{D}$ éch. $200 \mu \mathrm{m} ; \mathrm{G}, \mathrm{L}$ éch. 100 $\mu \mathrm{m} ; \mathrm{E}, \mathrm{F}, \mathrm{H}, \mathrm{I}, \mathrm{J}$ éch. $50 \mu \mathrm{m} ; \mathrm{M}$, éch. $25 \mu \mathrm{m}$; N et $\mathrm{O}$, à main levée). 


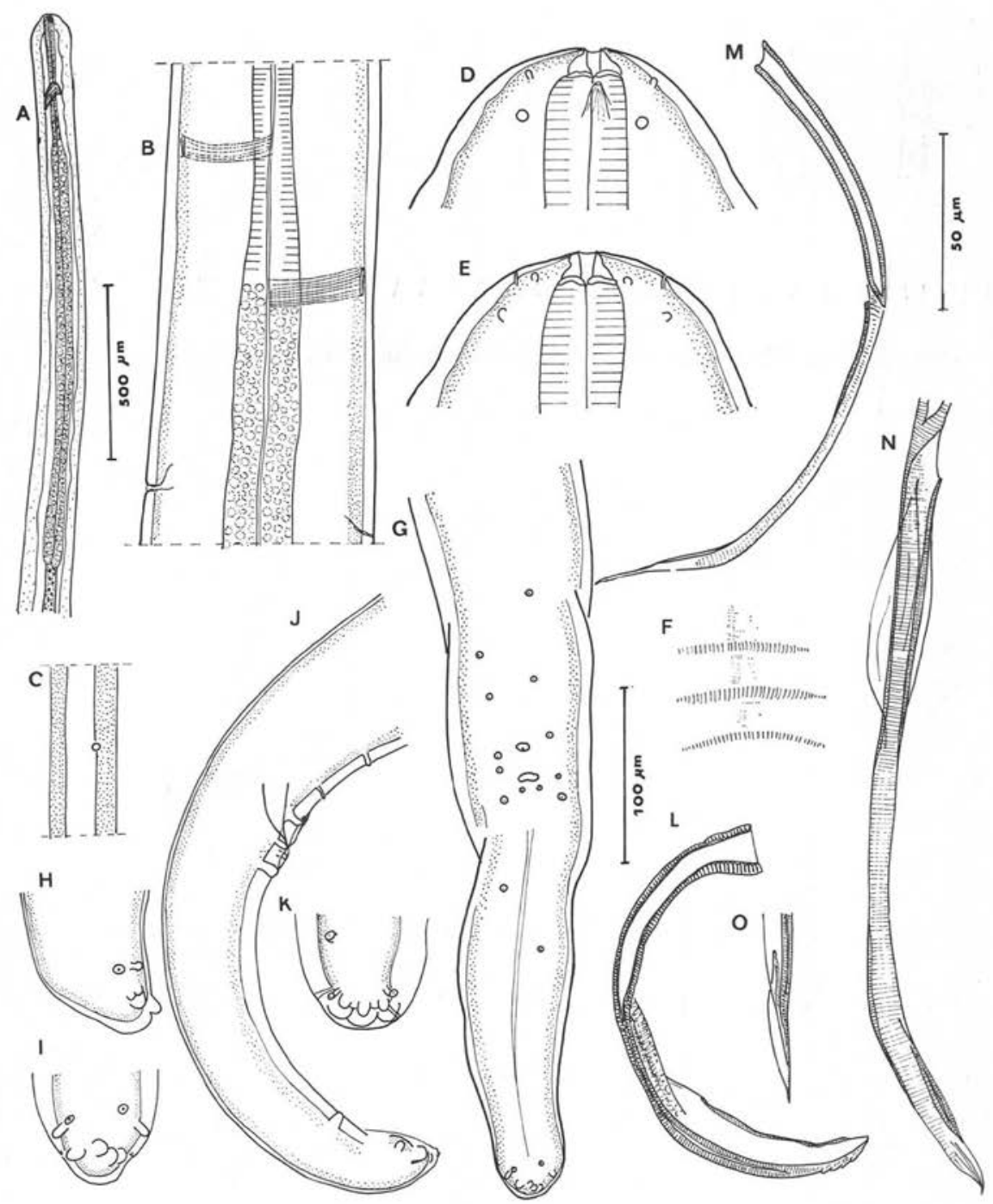

FIG. 4. - Acanthocheilonema pachycephalum, ô; A : région antérieure, vue latérale ; B : position des deirides, vue médiane; $\mathrm{C}$ : une deiride vue de face; $\mathrm{D}$ et $\mathrm{E}$ : tête, vue latérale et médiane ; $\mathrm{F}$ : détail de l'area rugosa, vue ventrale; $\mathrm{G}$ : queue d'I $\hat{\sigma}$, vue ventrale ; $\mathrm{H}$ et I : idem, extrémité caudale, vues latérale et ventrale ; J : queue de l'autre $\widehat{\sigma}$, vue latérale ; $\mathrm{K}$ : idem, extrémité caudale, vue ventrale; $\mathrm{L}$ : spicule droit, vue latérale; $\mathrm{M}$ : spicule gauche vue latérale ; $\mathrm{N}$ : structure de la lame du spicule gauche; O : pointe du spicule gauche; (A, éch. $500 \mu \mathrm{m}$; B, G, J éch. roo $\mu \mathrm{m}$; C, D, E, F, H, I, K, L, M, N, O éch. $50 \mu \mathrm{m})$. 
de $1520 \mu \mathrm{m}$ avec portion musculaire longue de $330 \mu \mathrm{m}$; area rugosa commençant à $800 \mu \mathrm{m}$ de l'extrémité caudale et longue de $2 \mathrm{~mm}$; spicule gauche long de $390 \mu \mathrm{m}$ (manche long de $150 \mu \mathrm{m}$ ) et spicule droit long de $170 \mu \mathrm{m}$ (manche long de $75 \mu \mathrm{m}$ ) ; queue longue de $265 \mu \mathrm{m}$; pointes caudales hautes de 6-7 $\mu \mathrm{m}$.

\section{Discussion}

Ces Filaires appartiennent clairement au genre Acanthocheilonema Cobbold, 1870 : capsule buccale bien formée (2 segments), œsophage glandulaire développé, deirides présentes.

Deux espèces ont été décrites en Afrique du Sud chez les petits Carnivores :

- A. setariosa (Monnig, 1927), parasite du tissu adipeux d'un Viverridé, Suricata suricatta Schr. : cette espèce diffère de nos spécimens par la taille plus grande des adultes, la vulve plus postérieure et la forme de l'extrémité caudale ornée par trois pointes allongées et coniques.

- A. pachycephalum (Ortlepp, 1961), parasite des veines hépatiques d'un Mustelidé, Ictonyx striatus (Perry) : la morphologie de cette espèce est identique à celle de nos spécimens à laquelle nous les identifions (même forme des tubercules caudaux, de la tête, même disposition des papilles caudales, etc.).

\section{IV - Cercopithifilaria degraaffi n. sp.}

\section{Matériel} Paris.

1 ㅇ holotype, 3 q et une région antérieure de + paratypes, no 54 KB, M.N.H.N.,

Hôte : Papio ursinus Kerr, en Afrique du Sud (Krüger Park, à Skukusa, octobre 1980); Filaires logées sous la peau (flanc et dos).

\section{Morphologie (fig. 5)}

Corps avec 3 renflements : un juste après la vulve, les deux autres situés respectivement à $3000 \mu \mathrm{m}$ et $7000 \mu \mathrm{m}$ de la vulve.

Tête trapézoïdale en vues médiane et latérale.

Papilles de la tête disposées suivant deux rectangles étirés latéralement, celui constitué par les quatre papilles céphaliques étant très postérieur (coupe apicale effectuée par erreur en avant de ces papilles) ; amphides au même niveau que les papilles labiales externes; deux papilles labiales externes latérales atrophiées situées près des phasmides.

Bouche petite et arrondie ; cavité buccale également petite, presque cylindrique, limitée en avant par le bord épaissi de la cuticule céphalique et en arrière par un anneau cuticulaire de très petite taille. 

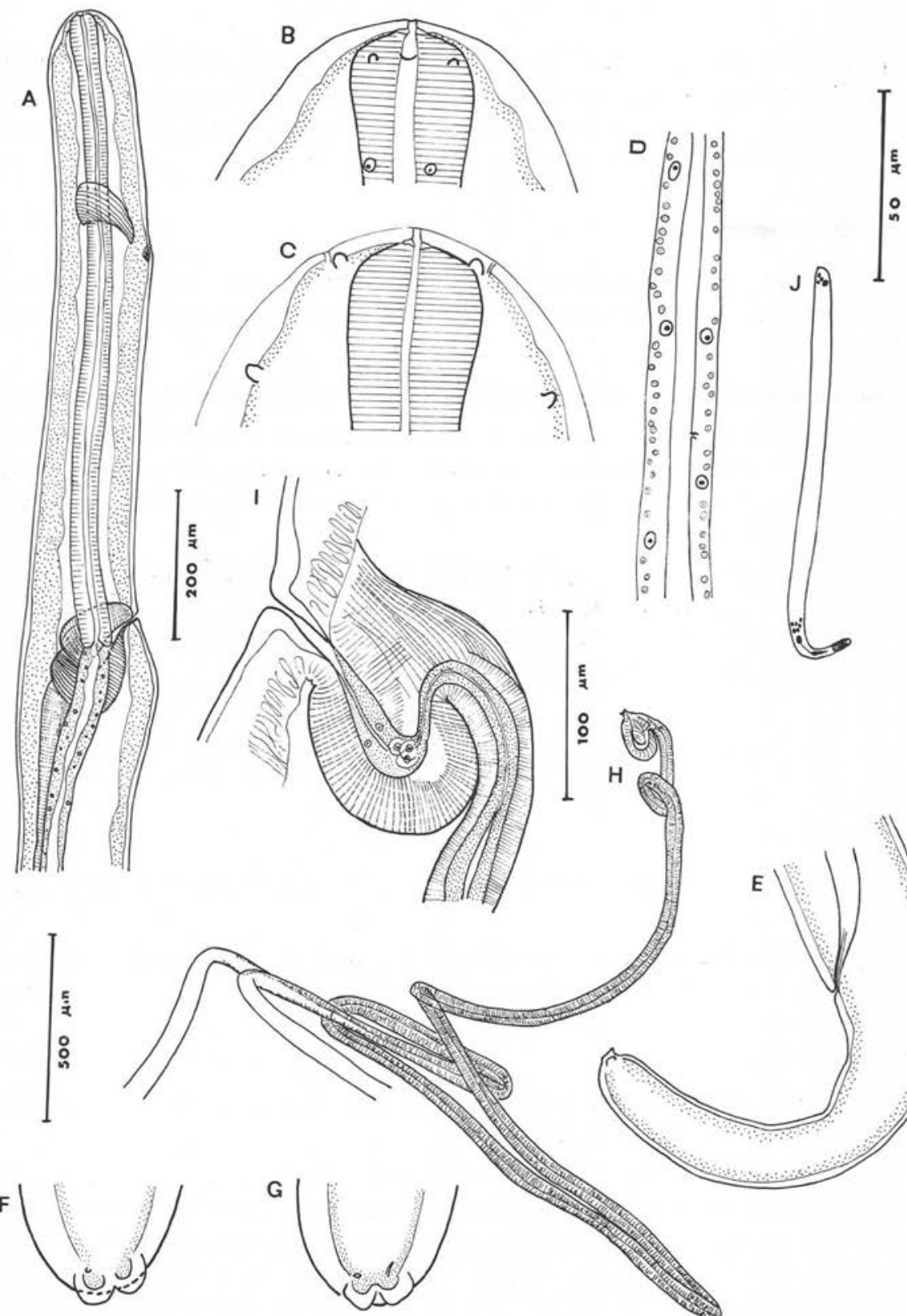

FIG. 5. - Cercopithifilaria degraaffi n. sp., 우 ; A : région antérieure, vue latérale ; B et C : tête, vues latérale et médiane ; $\mathrm{D}$ : détail de la structure de l'œsophage; $\mathrm{E}$ : région caudale, vue latérale; $\mathrm{F}$ et $\mathrm{G}$ : extrémités caudales en vue ventrale de 2 우 $\mathrm{H}$ : ovéjecteur; $\mathrm{I}$ : vagin; $\mathrm{J}$ : microfilaire utérine (A, éch. $200 \mu \mathrm{m} ; \mathrm{B}, \mathrm{C}$, et $\mathrm{E}, \mathrm{F}, \mathrm{G}, \mathrm{J}$, éch. $50 \mu \mathrm{m}$; I et E, éch. roo $\mu \mathrm{m} ; \mathrm{H}$, éch. $500 \mu \mathrm{m})$. 
Esophage d'épaisseur uniforme à portion glandulaire non différenciée* et à lumière aplatie.

Ovéjecteur long avec vagin complexe comprenant un court segment distal droit à cuticule épaisse suivi d'une portion en S tapissée par l'épithélium. Cette portion comprend une chambre allongée suivie par un tube étroit et coudé ; entre les deux se trouvent quatre cellules arrondies qui font office de sphincter.

Queue courbée ventralement; deux languettes subterminales presque ventrales, aussi larges que longues, à apex arrondi ou tronqué.

Microfilaire sans corps interne, à tête ronde; petit espace céphalique, crochet exigu, noyaux somatiques serrés ; pointe caudale avec un long noyau effilé suivi d'un noyau terminal triangulaire ou ovale. En arrière de la pointe caudale se trouvent trois à cinq paires de granules réfringents, disposés en deux rangées régulières terminées par un plus gros granule. Ces granules maintenus à la queue de la microfilaire, démontrent l'existence d'une gaine.

\section{Dimensions}

ㅇ holotype : corps long de $60 \mathrm{~mm}$ et large de $210 \mu \mathrm{m}$; anneaux nerveux à $260 \mu \mathrm{m}$ de l'apex; cavité buccale haute de $5 \mu \mathrm{m}$, anneau buccal haut de $2 \mu \mathrm{m}$ et à paroi large de $4 \mu \mathrm{m}$ au maximum; œsophage long de $860 \mu \mathrm{m}$; vulve à $830 \mu \mathrm{m}$ de l'apex, vagin long de $140 \mu \mathrm{m}$ et large de $95 \mu \mathrm{m}$, portion impaire de l'ovéjecteur longue de $5900 \mu \mathrm{m}$; queue longue de $315 \mu \mathrm{m}$ et languettes caudales longues de $6 \mu \mathrm{m}$.

Une $q$ paratype : corps long de $49 \mathrm{~mm}$ et large de $150 \mu \mathrm{m}$; rectangle des papilles céphaliques long de $82 \mu \mathrm{m}$ et large de $18 \mu \mathrm{m}$; anneau nerveux à $270 \mu \mathrm{m}$ de l'apex ; œsophage long de $1080 \mu \mathrm{m}$; vulve à $840 \mu \mathrm{m}$ de l'apex; queue longue de $240 \mu \mathrm{m}$.

Microfilaire : corps long de 118 à $125 \mu \mathrm{m}$ et large de $5,5 \mu \mathrm{m}$; espace céphalique long de $2 \mu \mathrm{m}$; pointe caudale longue de $12 \mu \mathrm{m}$; espace occupé par les granules réfringents long de $8 \mu \mathrm{m}$.

\section{Discussion}

Chez les singes en Afrique, hormis les Tetrapetalonema des Anthropoïdes et de l'Homme, on ne connaissait pas de représentants du groupe Dipetalonema jusqu'à ces dernières années.

Récemment un Dipetalonema sous-cutané a été trouvé chez un Babouin, Papio anubis au Kenya : Dipetalonema (Ceropithifilaria) kenyensis Eberhard, 1980. Il s'agit d'une espèce évoluée, caractérisée par le petit anneau cuticulaire préœsophagien, l'œsophage non divisé et court, les papilles caudales du ô en nombre réduit, rassemblées près du cloaque et le spicule droit large et à manche réduit.

Nous proposons d'élever le sous-genre au niveau générique.

* En fait, les noyaux glandulaires, très petits, sont visibles au fort grossissement sur les $2 / 3$ postérieurs de l'osophage ( fig. $s D$ ). Deux pores glandulaires débouchant dans la lumière œsophagienne sont également visibles. 
Nos spécimens sont proches de cette espèce mais s'en distinguent par l'œsophage plus long et la présence, chez la microfilaire, de granules réfringents caudaux.

Ils constituent une espèce nouvelle que nous dédions à $M$. de Graaff, et nommons Cercopithifilaria degraaffi $\mathrm{n}$. sp.

\section{V - Cercopithifilaria cephalophi n. sp.}

\section{Matériel}

1 ㅇ holotype no 84 DS, M.N.H.N. Paris.

Hôte : Cephalophus dorsalis Gray, au Gabon (Belinga, août 1981) ; Filaire logée sous la peau d'une cuisse ; microfilaires dermique.

\section{Morphologie (fig. 6)}

Corps avec un léger épaississement en arrière de la vulve.

Tête arrondie, suivie par un très léger rétrécissement du corps. Papilles bien saillantes, les quatre labiales externes disposées suivant un rectangle étiré latéralement et les quatre céphaliques presque suivant un carré. Cavité buccale petite, limitée en avant par la cuticule céphalique et en arrière par un petit anneau cuticulaire.

Esophage sans différenciation musculoglandulaire visible* d'épaisseur régulière et à lumière aplatie.

Ovéjecteur avec vagin complexe et cellules épithéliales gonflées formant sphincter ; l'ovéjecteur s'évase et sa paroi s'amincit bien avant la division en deux utérus.

Queue effilée, légèrement courbée ventralement, terminée par deux pointes coniques à extrémité obtuse ; à la base des pointes se trouvent latéralement les phasmides et dorsalement une petite saillie cuticulaire.

Microfilaire à corps peu atténué en avant, effilé en arrière ; tête arrondie à crochet très petit ; ailes latérales bien développées ; espace céphalique grand ; noyaux somatiques espacés ; pas de corps interne ; portion caudale anucléée longue et relativement épaisse ; gaine non observée.

\section{Dimensions}

ㅇ holotype : corps long de $29 \mathrm{~mm}$ et large de $115 \mu \mathrm{m}$; anneau nerveux à $190 \mu \mathrm{m}$ de l'apex; cavité buccale haute de $5,5 \mathrm{~mm}$ et anneau buccal haut de $2 \mu \mathrm{m}$, à paroi large de $5 \mu \mathrm{m}$; œsophage long de $1230 \mu \mathrm{m}$; vulve à $850 \mu \mathrm{m}$ de l'apex, fusion des utérus à $1200 \mu \mathrm{m}$ de la vulve ; queue longue de $200 \mu \mathrm{m}$ et pointes caudales longues de $10-11 \mu \mathrm{m}$.

Microfilaires longues de 218 à $232 \mu \mathrm{m}$ et larges de $5,5 \mu \mathrm{m}$ en vue médiane $(4,5 \mu \mathrm{m}$ sans les ailes latérales) ; espace céphalique long de 8-10 $\mu \mathrm{m}$; portion caudale anucléée longue de $25 \mu \mathrm{m}$.

* Comme pour l'espèce précédente, noyaux et pores glandulaires sont présents, mais visibles seulement à un fort grossissement ( $f$ g. $6 \mathrm{E}$ ). 


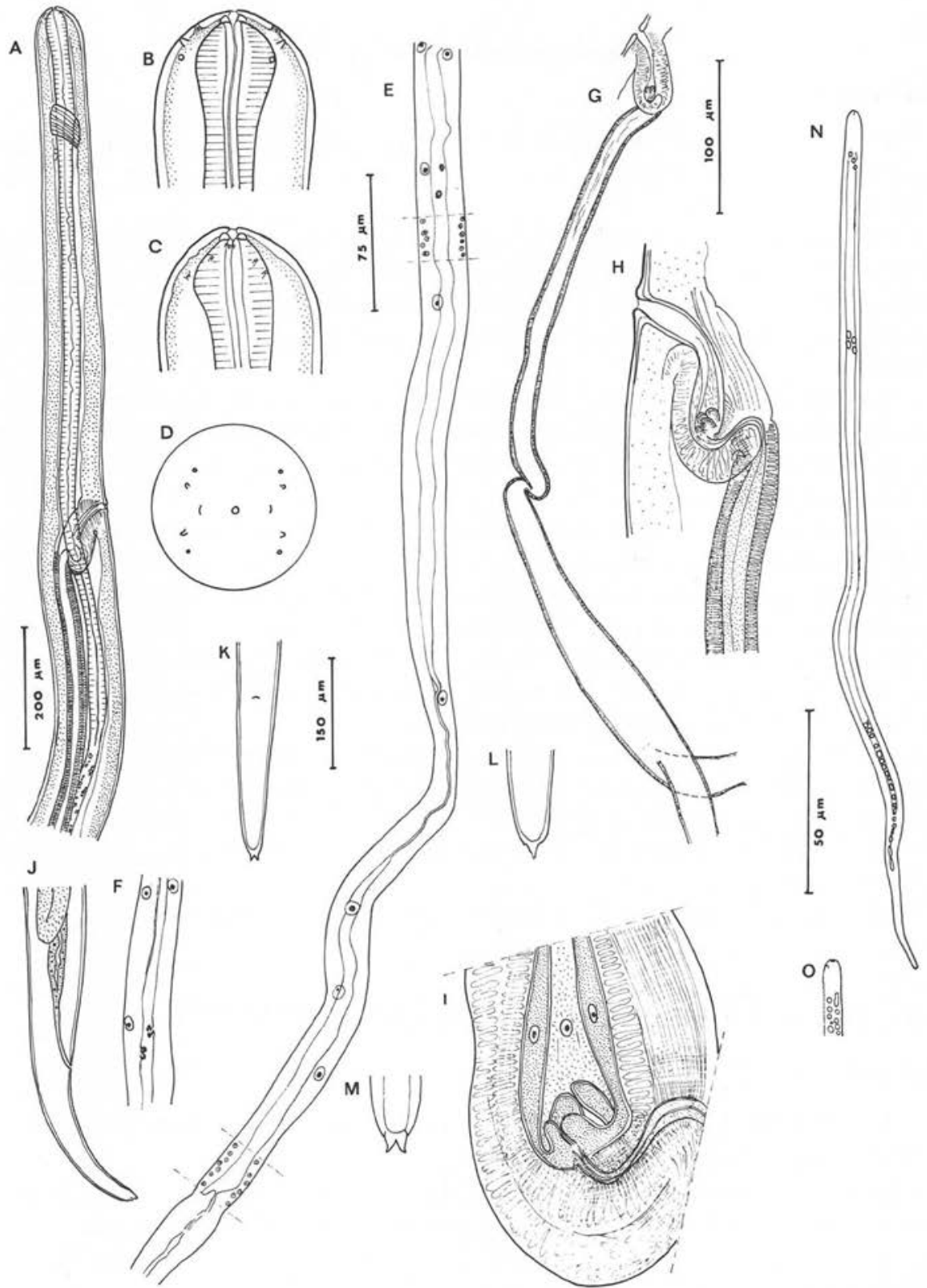

FIG. 6. - Cercopithifilaria cephalophi n. sp., 우 ; : région antérieure, vue latérale ; B, C, : tête, vues médiane et latérale ; D : reconstitution théorique d'une vue apicale ; $\mathrm{E}$ : structure de la moitié postérieure de l'œsophage (les petits noyaux glandulaires sont représentés seulement entre les traits en tirets ; F : idem, pores œsophagiens vus de profil; G : ovéjecteur ; H : vagin; I : détail du sphincter; J et $\mathrm{K}$ : queue, vues latérale et médiane ; L et M : extrémité caudale, vues latérale et médiane; $\mathrm{N}$ : microfilaire utérine ; $\mathrm{O}: i d e m$, tête, crochet vu de profil (A, G, éch. $200 \mu \mathrm{m} ; \mathrm{B}, \mathrm{C}, \mathrm{E}, \mathrm{F}, \mathrm{H}$ et M, éch. $100 \mu \mathrm{m}$; I, éch. $30 \mu \mathrm{m} ; \mathrm{J}, \mathrm{K}$, éch. $75 \mu \mathrm{m} ; \mathrm{N}$, $\mathrm{O}$, éch. $50 \mu \mathrm{m})$. 


\section{Discussion}

Plusieurs espèces de Dipetalonema sont déjà connues chez les Bovidés africains.

Comme il a été montré plus haut, ces Filaires ne peuvent plus être placées dans le taxon Loxodonto filaria.

Par plusieurs caractères évolués (capsule buccale très petite, œsophage court non divisé et grêle, spicule droit à manche très réduit) elles sont proches des Cercopithifilaria de Singes africains mais le nombre et la disposition des papilles caudales du $\widehat{\sigma}$ sont beaucoup plus proches du plan Spiruride primitif que chez les espèces parasites de Singes.

Il semble toutefois justifier de placer dans le même genre ces deux groupes de parasites car ils pourraient représenter deux degrés évolutifs d'une même petite lignée dérivée d'Acanthocheilonema, un peu plus primitive chez les Bovidés, un peu plus évoluée chez les Singes.

A ce groupe de Cercopithifilaria parasite de Bovidés africains, se rattache morphologiquement l'espèce parasite d'un Cervidé européen, C. rugosicauda (Böhm et Supperer, 1953) n. comb., redécrite par Meszaros et Sugar (1976). Certaines structures que nous avons observées sur 2 femelles de cette espèce (lot $500 \mathrm{JR}$, Capreolus capreolus, Tubingen, Allemagne Fédérale), comme la capsule buccale, l'œsophage et la portion distale impaire des utérus ( fig. 9), sont également bien compatibles avec les Cercopithifilaria des Bovidés africains.

La Filaire de Cephalophus dorsalis est distincte, d'une part des trois espèces parasites des Bovidés africains, C. ruandae (Fain et Herin, 1955), C. dermicola (Fain, 1977) et C. faini (Chabaud, Landau et Petit, 1978), et, d'autre part de C. rugosicauda, par l'œesophage plus long et par l'extrémité caudale ornée par deux simples pointes coniques terminales.

Elle constitue une nouvelle espèce, Cercopithifilaria cephalophi n. sp.

\section{VI - Cercopithifilaria gabonensis n. sp.}

\section{Matériel}

1 o holotype, 1 ô allotype, 2 fragments antérieurs et 2 fragments postérieurs de ㅇ, no 89 DS, et coll. M.N.H.N., Paris.

Hôte : Atherurus africanus Gray, Makokou, Gabon, août 1981 ; Filaires localisées dans le tissu conjonctif sous-cutané (aines, aisselles) ; microfilaires dermiques.

\section{Morphologie ( fig. 7 et 8 )}

Corps à cuticule épaissie dans le plan latéral. Tête arrondie suivie d'un cou peu marqué.

Six petites papilles visibles, plus les amphides, disposées suivant la figure $7 \mathrm{~B}$ et $D$. Bouche presque ronde. Cavité buccale limitée en avant par la cuticule céphalique épaisse et en arrière par un anneau cuticulaire 2-3 fois plus mince que la cuticule 


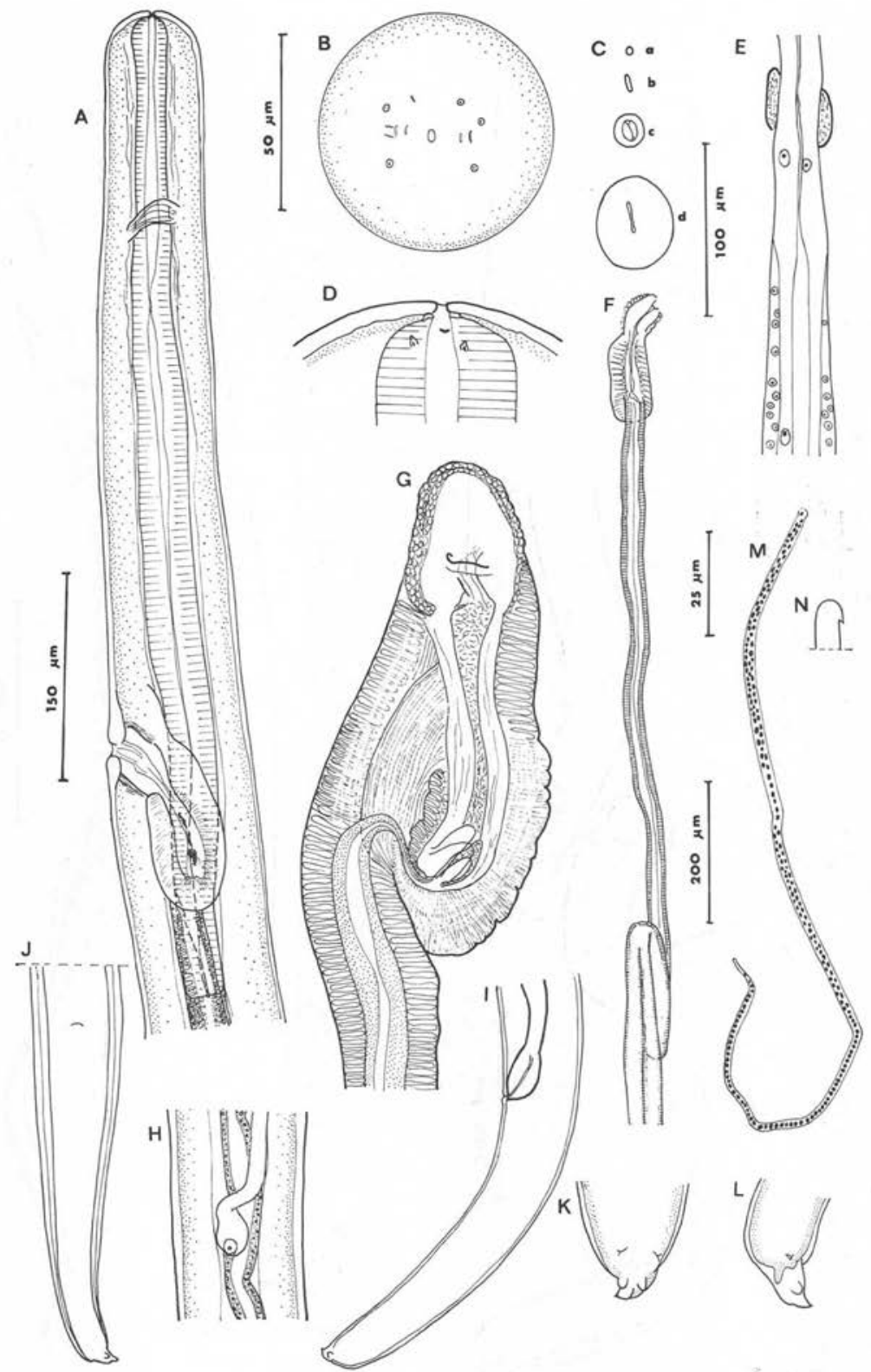

FIG. 7. - Cercopithifilaria gabonensis n. §p., ㅇ; A : région antérieure, vue latérale; B : tête, vue apicale; $\mathrm{C}$ : bouche (a) puis coupes optiques à 2 niveaux de la capsule buccale (b, c), et dans la région antérieure de l'œsophage (d) ; D : tête, vue latérale ; E : structure de l'œsophage dans la région de l'anneau nerveux ; F : ovéjecteur ; $\mathrm{G}$ : vagin, vue ventrale ; $\mathrm{H}$ : apex d'un ovaire; I et J : queue, vues latérale et ventrale; $\mathrm{K}$ et $\mathrm{L}$ : extrémité caudale, vues ventrale et latérale; M : microfilaire utérine; $\mathrm{N}$ : idem, tête, crochet vu de profil (A, H, I, J éch. I $50 \mu \mathrm{m} ; \mathrm{B}, \mathrm{C}, \mathrm{D}, \mathrm{G}, \mathrm{K}, \mathrm{L}$ éch $50 \mu \mathrm{m} ; \mathrm{E}$ éch. $100 \mu \mathrm{m} ; \mathrm{F}$, éch. $200 \mu \mathrm{m} ; \mathrm{M}$, éch. $25 \mu \mathrm{m} ; \mathrm{N}$, main levée). 


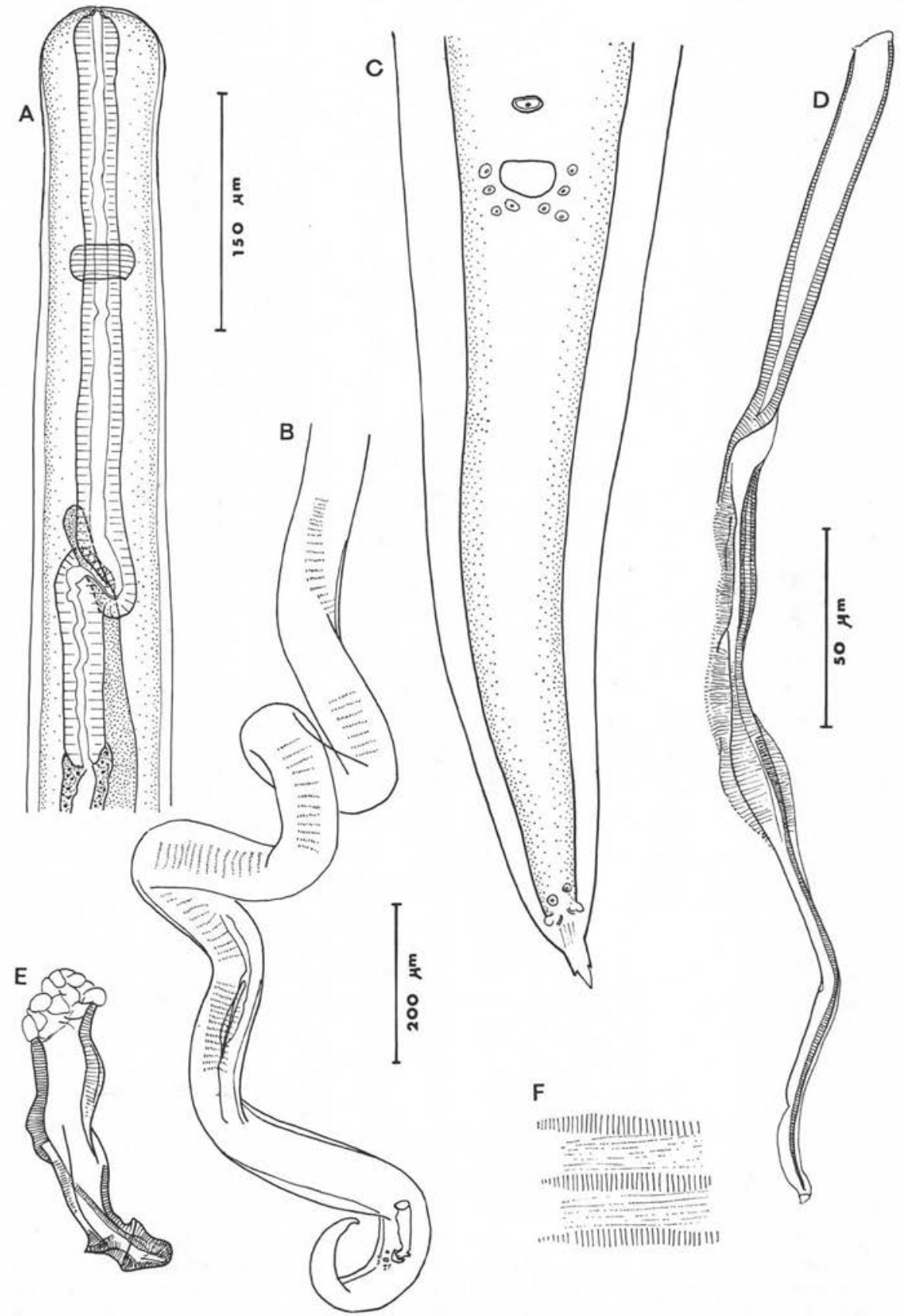

Fig. 8. - Cercopithifilaria gabonensis $\mathrm{n}$. sp., ôં; A : région antérieure, vue médiane; B : région postérieure ; $\mathrm{C}$ : queue, vue ventrale ; D : spicule gauche disséqué ; $\mathrm{E}$ : spicule droit disséqué, vue latérale; F : détail de l'area rugosa; (A, éch. I50 $\mu \mathrm{m} ; \mathrm{B}$, éch. $200 \mu \mathrm{m} ; \mathrm{C}, \mathrm{D}, \mathrm{E}, \mathrm{F}$, éch. $50 \mu \mathrm{m})$. 
céphalique ; la partie antérieure de la cavité buccale est aplatie latéralement, l'anneau préœsophagien est légèrement étiré latéralement. Esophage à lumière aplatie, sur toute la longueur de l'organe ; différenciation glandulaire visible seulement au fort grossissement (fig. $7 \mathrm{~A}$ et $\mathrm{E}$ ). Dans les deux sexes, queue brusquement rétrécie en cône, près de l'extrémité ; pointe terminale du cône ornée d'un mucron et, à mi-hauteur du cône, présence de deux languettes latérales, très petites chez le $\delta$.

Femelle: vulve un peu en avant de la fin de l'œsophage, située sur un léger renflement ventral du corps ; cuticule épaisse autour de la vulve. Vagin puissant comprenant, d'avant en arrière, un tube cuticulaire court à lumière étroite, puis une chambre allongée tapissée de cuticule épaisse et limitée postérieurement par trois languettes cuticulaires formant sphincter, enfin un tube très étroit et court en forme d'S et sans cuticule ; ovéjecteur dirigé vers l'arrière. Ovaires à apex arrondi, naissant dans la région postérieure du corps. Queue longue et fine, courbée ventralement à son extrémité ; phasmides situées un peu en avant du cône terminal.

Microfilaires utérines : corps très fin, sans gaine, à crochet céphalique assez long, espace céphalique bien marqué ; pas de corps interne ; queue pointue nucléée.

Mâle : testicule à apex arrondi, naissant à la hauteur de l'œsophage. Région postérieure spiralée (2-3 tours) ; queue à cuticule fortement épaissie latéralement ; grand orifice cloacal; papilles peu nombreuses : un groupe cloacal (4 paires plus une impaire précloacale) et un groupe subterminal (2 paires et les phasmides). Spicule droit bien cuticularisé, mais sans manche étroit, renforcé dans la région distale par un fort épaississement spiralé formant 2 tours ( 2 saillies bien visibles sur la face dorsale). Spicule gauche assez grèle avec une lame complexe, comprenant une moitié antérieure large, en gouttière et à rides transversales et une moitié postérieure plus étroite dont l'axe cuticularisé est bordé par deux ailes membraneuses.

\section{Dimensions}

† holotype : corps long de $95 \mathrm{~mm}$ et large de $140 \mu \mathrm{m}$; anneau nerveux et pore excréteur à 160 et $215 \mu \mathrm{m}$ de l'apex; œesophage long de $730 \mu \mathrm{m}$; vulve à $580 \mu \mathrm{m}$ de l'apex, sphincter long de $180 \mu \mathrm{m}$ et large de $65 \mu \mathrm{m}$; portion impaire de l'ovéjecteur longue de $1300 \mu \mathrm{m}$; queue longue de $280 \mu \mathrm{m}$.

Fragments $q$ : corps large de $150 \mu \mathrm{m}$; anneau nerveux à $185 \mu \mathrm{m}$ de l'apex ; œsophage long de $890 \mu \mathrm{m}$ (portion musculaire longue de $300 \mu \mathrm{m}$ ); vulve à $660 \mu \mathrm{m}$ de l'apex, sphincter long de $165 \mu \mathrm{m}$ et large de $70 \mu \mathrm{m}$; queue longue de $340 \mu \mathrm{m}$, avec cône terminal de $17 \mu \mathrm{m}$, languettes latérales de $5 \mu \mathrm{m}$ et pointe terminale de $6 \mu \mathrm{m}$; ovaires à 1200 et $2300 \mu \mathrm{m}$ de l'extrémité caudale.

Microfilaires utérines (fixées à l'alcool chaud) : corps long de 200-222 $\mu \mathrm{m}$ sur $2,5 \mu \mathrm{m}$ de large ; espace céphalique long de $3 \mu \mathrm{m}$; région du pore excréteur à $70 \mu \mathrm{m}$ de l'apex.

$\delta$ allotype : corps long de $56 \mathrm{~mm}$ et large de $140 \mu \mathrm{m}$; anneau nerveux et naissance du testicule à 200 et $400 \mu \mathrm{m}$ de la tête ; œsophage long de $650 \mu \mathrm{m}$; queue longue de 
$250 \mu \mathrm{m}$ avec cône terminal long de $17 \mu \mathrm{m}$; spicule gauche long de $380 \mu \mathrm{m}$ (manche long de $140 \mu \mathrm{m}$ ), spicule droit long de $100 \mu \mathrm{m}$ (crochet le plus antérieur à $20 \mu \mathrm{m}$ de l'extrémité distale) ; area rugosa s'étendant de 700 à $2200 \mu \mathrm{m}$ de l'extrémité caudale ; rides cuticulaires hautes de 5-6 $\mu \mathrm{m}$; espacement de 7-9 $\mu \mathrm{m}$ entre deux bandes de rides.

\section{Discussion}

Cette Filaire d'Athérure a tout un ensemble de caractères évolués (capsule buccale très petite, œsophage court, non divisé et à lumière aplatie, papilles caudales en nombre réduit et groupées près du cloaque) et diverses structures (sphincter formé de 3-4 languettes cellulaires; spicule droit trapu sans manche distinct) qui la situent dans le genre Cercopithifilaria.

Ces spécimens s'opposent à tous les Cercopithifilaria qui parasitent les Artiodactyles par la grande réduction des papilles caudales, l'extrémité distale du spicule droit orné d'une côte spiralée à 2 tours.

Ils s'opposent également aux deux espèces des Singes africains par la forme de l'extrémité caudale de la , par la microfilaire deux fois plus longue et plus mince et, dans le cas de $C$. kenyensis dont le ô est connu, par la structure plus complexe des spicules gauche et droit.

Elle constitue donc une espèce nouvelle, Cercopithifilaria gabonensis n. sp.

\section{VII - Cercopithifilaria didelphis}

(Esslinger et Smith, 1979) n. comb.

\section{Matériel}

Plusieurs ô et ? , lot 184 EJ, M.N.H.N. Paris.

Hôte : Didelphis marsupialis L., Pichinde, près de Cali, état de Valle, Colombie, 21-3-80 ; Filaires logées dans le tissu conjonctif sous la scapula ; microfilaires dermiques.

\section{Morphologie (fig. 9 I à $O$ et $10 \mathrm{I}$ )}

L'espèce est bien décrite et nous analysons seulement quelques caractères pour les comparer à ceux des autres Cercopithifilaria :

- L'anneau préœsophagien est inexistant sur nos spécimens; l'œsophage arrive directement au contact de la cuticule céphalique, très amincie près de la bouche.

- L'œsophage a une lumière aplatie.

- La structure du fourreau épithélio-musculaire est particulière : les cordes latérales sont très épaisses et 2 crêtes cuticulaires internes les soutiennent.

- Les spicules sont extrêmement fins.

- Les papilles situées près du cloaque ont la disposition figurée par Esslinger et Smith ; il existe en outre une double paire de papilles près de l'extrémité caudale. 

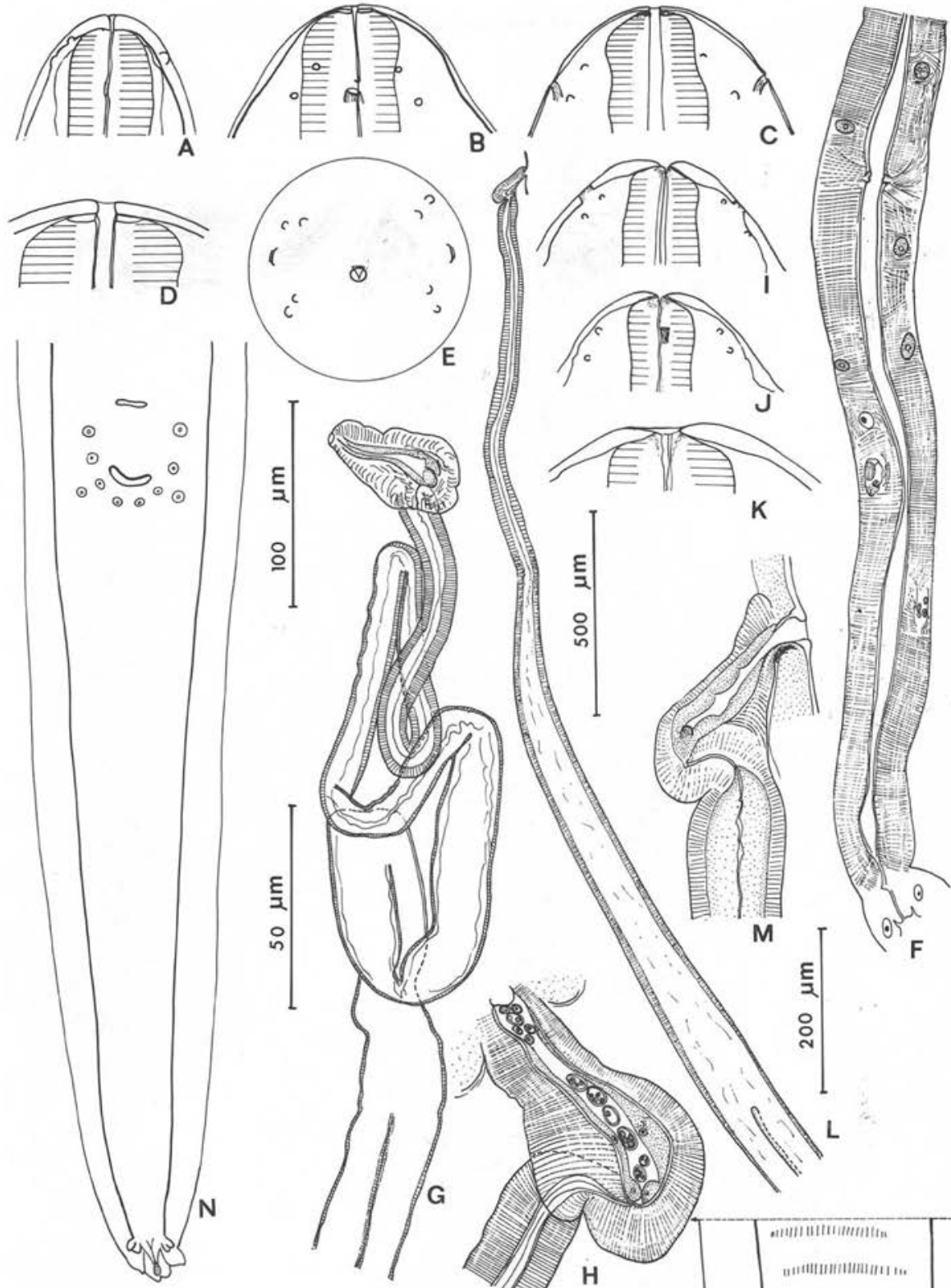

K
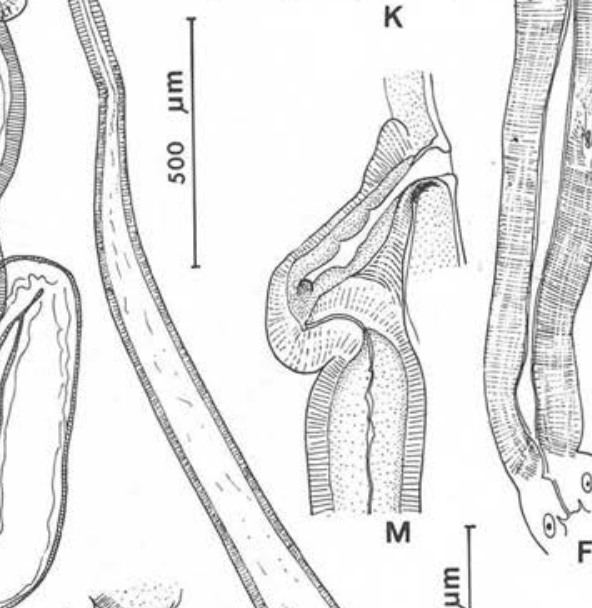

Fig. 9. - A : Dipetalonema okapiae, tête + ; $\mathrm{B}$ à $\mathrm{H}$ : Cercopithifilaria rugosicauda ; B et $\mathrm{C}$ : tête, vues latérale et médiane ; D : cavité buccale ; E : tête, vue apicale ; F : structure de l'œsophage (les fibrilles musculaires radiaires sont puissantes) ; $\mathrm{G}$ : ovéjecteur; $\mathrm{H}$ : vagin ; I à $\mathrm{O}: C$. didelphis; I et $\mathrm{J}$ : tête, vues médiane et latérale; $\mathrm{K}$ : cavité buccale; $\mathrm{L}$ : ovéjecteur; $\mathrm{M}$ : vagin; $\mathrm{N}$ : queue $\hat{\mathrm{O}}$, vue ventrale; $\mathrm{O}$ : area rugosa, détail. (A, B, C, E, F, H, I, J, M éch. $100 \mu \mathrm{m}$ : D, K, N, O, éch. $50 \mu \mathrm{m}, \mathrm{G}$ éch. $500 \mu \mathrm{m}, \mathrm{L}$ éch. $200 \mu \mathrm{m})$. 

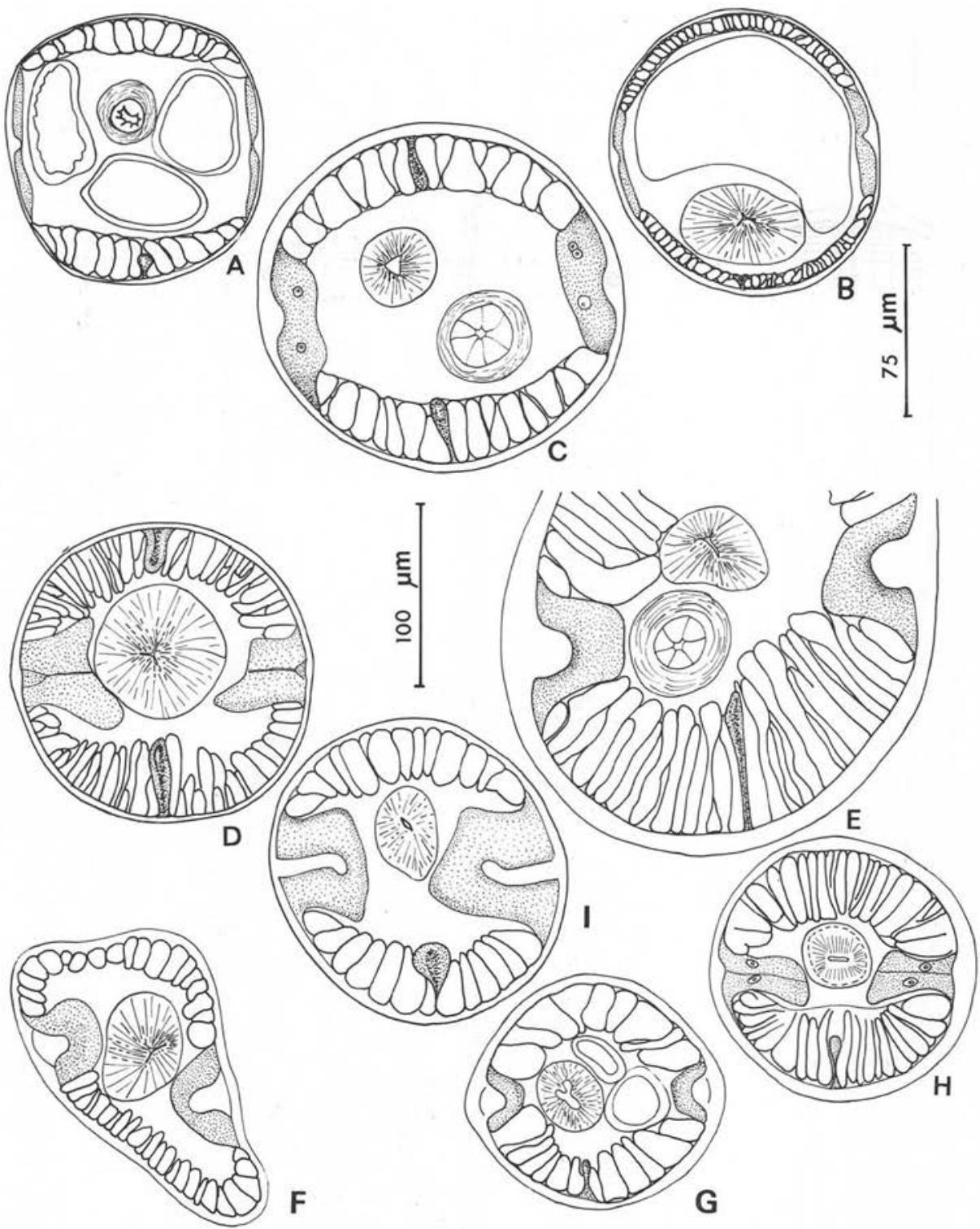

FIG. ro. - Section transversale du corps, dans la moitié postérieure de l'œsophage (sauf pour la fig. A) ; A : Litomosa sp., Miniopterus schreibersi, grotte de Pouade, Pyrénnées Orientales, France, 19.9.48 (coupe postœsophagienne); B : Breinlia (B.) spratti Bain et coll., 1979, lot 165 JE, Callosciurus notatus, Malaisie; C : Skrijabinofilaria skrjabini Travassos, 1925, lot 39I JE, Metachirops oppossum, Guyane Française ; D : Orihelia sp., lot 689 TW, Euphractus sexinxtus, Brésil ; E : Dipetalonema gracile (Rudolphi, I809), lot 227 NE, Saimiri sciureus, Guyane Française; F : Acanthocheilonema viteae (Krepkogorskaya, I953), lot 89 JN, cycle expérimental chez le hamster; G : Molinema dessetae Bain, 1973, lot 8I GK, cycle expérimental chez Proechimys oris ; $\mathrm{H}$ : Cercopithifilaria degraaffi n. sp. ; I : Cercopithifilaria didelphis (Esslinger et Smith, 1979), lot I84 EJ, Didelphis marsupialis, Colombie. (A, B, C, D, E, G, $\mathrm{H}, \mathrm{I}$ éch. $100 \mu \mathrm{m} ; \mathrm{F}$, éch. $75 \mu \mathrm{m})$. 


\section{Dimensions}

Une $ᄋ$ : corps long de $56 \mathrm{~mm}$, large de $160 \mu \mathrm{m}$; anneau nerveux et vulve à $180 \mu \mathrm{m}$ et $1060 \mu \mathrm{m}$ de l'apex; œsophage large de $720 \mu \mathrm{m}$ avec portion glandulaire longue de $320 \mu \mathrm{m}$, ovéjecteur long de $2600 \mu \mathrm{m}$; queue longue de $320 \mu \mathrm{m}$; microfilaires utérines longues de 87-88 $\mu \mathrm{m}$ et larges de $4 \mu \mathrm{m}$.

Un $\sigma^{~}$ : corps long de $29 \mathrm{~mm}$, large de $90 \mu \mathrm{m}$, anneau nerveux à $210 \mu \mathrm{m}$ de l'apex ; œsophage long de $700 \mu \mathrm{m}$ avec portion glandulaire longue de $450 \mu \mathrm{m}$; queue longue de $195 \mu \mathrm{m}$; spicule gauche long de $195 \mu \mathrm{m}$; spicule droit long de $54 \mu \mathrm{m}$.

\section{Discussion}

Notre matériel s'identifie à $A$. didelphis Esslinger et Smith, 1979.

L'espèce a été considérée comme un Acanthocheilonema par ces auteurs. Effectivement, elle n'a pas d'affinités avec les formes typiquement néotropicales mais se rapproche des Acanthocheilonema spécialisés, c'est-à-dire de Cercopithifilaria (œsophage légèrement divisé mais très court, papilles caudales en nombre très réduit, spicule droit sans manche distinct).

Un des éléments les plus remarquables de la morphologie est le profond renforcement cuticulaire invaginé à l'intérieur des cordes latérales. Pour tenter de comprendre la signification de ce caractère, des coupes transversales ont été effectuées chez des espèces variées (cf. liste des espèces, fig. 10).

Litomosa et Breinlia ont des cordes latérales plates avec un renforcement cuticulaire faible ; les autres espèces ont des cordes latérales généralement assez puissantes avec un renforcement cuticulaire d'importance variable sans que nous puissions mettre en évidence de corrélations avec la taille du corps ou avec la place zoologique de l'espèce.

Le caractère fourni par les cordes latérales de $C$. didelphis ainsi que la perte totale de la capsule buccale, sont des éléments morphologiques hyperspécialisés qui ne se retrouvent pas chez les autres espèces néotropicales.

Nous supposons donc que le parasitisme des Didelphis par cette espèce s'est effectué tardivement et en zone néarctique, ce qui expliquerait les analogies entre la Filaire des Didelphis et une Filaire de Lièvre canadien, en cours de description, dont nous avons eu connaissance grâce à l'amabilité du Dr. C. Bartlett.

\section{Conclusions générales}

Le nombre d'espèces connues dans la " lignée Dipetalonema » s'est largement accru au cours des récentes années.

Il devient nécessaire de les séparer en groupes distincts.

\section{I - Espèces australiennes}

L'area rugosa est constituée de petites rugosités irrégulièrement dispersées : genre Breinlia ; sous-genres Breinlia Yorke et Maplestone, 1926 et Johnstonema Yeh, 1957. 
Cet ensemble est interprété comme un groupe australien parasite de Marsupiaux ayant migré plus récemment en Asie (région orientale) chez différents Mammifères (Lémuriens-Rongeurs).

\section{II - Espèces paléoendémiques d'Amérique du Sud}

a) Espèce relique avec une capsule buccale ayant 3 segments distincts : genre Skrjabinofilaria, parasite de Didelphidés.

b) Espèces plus modernes, distinctes des autres Filaires de la "lignée Dipetalonema » par l'ensemble des caractères suivants : œsophage divisé, papilles de la tête très étirées latéralement, capsule buccale bien cuticularisée, 2 grands pétales caudaux dans les 2 sexes et microfilaires à gaine. On y trouve :

- des formes parasites de Xénarthres Dasypodidés : genres : Orihelia Chabaud et Bain, 1976, primitif, et Dasypafilaria Eberhard, 1982, qui semble dériver du genre précédent par réduction des papilles caudales et de la lame du spicule gauche.

- des formes parasites de Primates Platyrhiniens constituant le genre Dipetalonema Diesing, 1861 sensu stricto.

L'ensemble de ce groupe est interprété comme isolé en région néotropicale depuis la fragmentation du Gondwana et donc comme paléoendémique.

Les Platyrhiniens se seraient contaminés à partir de parasites préexistant dans ce continent.

\section{III - Espèces rattachables au groupe Tetrapetalonema}

Un troisième groupe paraît avoir évolué isolément dans le phylum InsectivorePrimate.

Ce groupe se distingue morphologiquement par la disparition totale de la capsule buccale, l'œsophage grêle et les 4 pétales caudaux.

Dans notre interprétation, il paraît avoir pris naissance (peut-être à partir d'Acanthocheilonema ?) en région orientale (genre Sandnema Chabaud et Bain, 1976), chez des Insectivores Soricoidea.

Le genre Tetrapetalonema Faust, 1935 se serait constitué chez les Tupaia en Orient, aurait évolué en Afrique en donnant le sous-genre Esslingeria Chabaud et Bain, 1976, et serait passé en Amérique du Sud à la fin de l'Éocène, en même temps que les Primates (cf. Hoffstetter, 1982) ; certaines formes hyperspécialisées (Filarissima Chabaud, 1974, parasite de Caviomorphes) pourraient en dériver.

\section{IV - Espèces rattachables au groupe Acanthocheilonema}

Le groupe particulièrement traité dans ce travail, paraît avoir son origine en Afrique chez les Insectivores et les Carnivores.

Nous envisageons son évolution de la façon suivante :

A - Le genre Acanthocheilonema. Il est primitif : capsule buccale bien cuticularisée, œsophage robuste et divisé, spicule droit avec manche bien formé et lamina en 
cuiller simple, rapport spiculaire de 2,1 à 2,9. Ce genre est parasite d'Insectivores et de Carnivores en région éthiopienne et paraît s'être répandu dans la région holarctique et la région orientale.

Les modifications taxonomiques que nous proposons sont les suivantes :

1 L'espèce-type de Chenofilaria, C. filaria Kou, 1958, parasite de Pholidote, avec son très long œsophage divisé et sa capsule buccale robuste (cf. Esslinger, 1966), peut être rattachée au genre Acanthocheilonema. Chenofilaria tombe en synonymie d'Acanthocheilonema.

2 D. grassii (Noe, 1907), parasite du Chien en Europe, par ses caractères évolués (œsophage très court, papilles caudales en nombre réduit, spicule droit trapu sans manche distinct ; cf. Noé, 1910) est exclu du genre Acanthocheilonema pour être placé dans le genre Cercopithifilaria.

3 Acanthocheilonema pricei (Vaz et Pereira, 1934) redécrit par Esslinger et Smith (1979), a bien, comme l'indiquent ces auteurs, la morphologie d'un véritable Acanthocheilonema sensu stricto.

L'espèce est connue de Didelphis marsupialis au Brésil.

Nous supposons donc que la capture s'est effectuée récemment, lors de la reprise des échanges entre l'Amérique du Nord et du Sud (Pliocène), à partir d'Acanthocheilonema de Carnivores de la zone néarctique.

B - Le genre Cercopithifilaria a été créé en sous-genre par Eberhard (1980) pour un parasite de Singe africain.

On peut y grouper toute une série d'espèces, qui paraissent être issues des Acanthocheilonema, dont elles se différencient par l'œsophage grêle et non divisé (ou, s'il est divisé, très court, inférieur à $1 \mathrm{~mm}$ ), la capsule buccale très petite, le spicule droit de forme trapue, sans manche distinct, les papilles caudales souvent en nombre réduit et rassemblées près du cloaque.

Nous y groupons :

1 La Filaire du chien, C. grassii, comme il est indiqué ci-dessus.

2 Les deux espèces parasites des singes Cercopithecidés, C. kenyensis Eberhard, 1980, espèce-type, et $C$. degraaffi n. sp.

3 Les espèces parasites des Bovidés africains, C. ruandae (Fain et Hérin, 1955), C. dermicola (Fain, 1977), C. faini (Chabaud et coll., 1978) et C. cephalophi n. sp., que nous avions cru pouvoir placer dans le taxon Loxodonto filaria, mais qui se séparent de l'espèce-type* par des caractères importants (capsule buccale présente, œsophage grêle, deirides absentes).

* L'espèce-type de Loxodontofilaria est L. loxodontis Berghe et Gillain, r939, dont L. asiatica n. sp. paraît très proche. Rien n'est connu sur les mâles, mais les femelles ont des éléments particuliers : absence d'anneau cuticulaire préœsophagien, œesophage robuste, présence de deirides. Sous cette forme provisoire, le genre comprend : L. loxodontis Berghe et Gillain, I939, L. gossi (Baylis, I923), L. asiatica n. sp. et $L$. hippopotami (Leiper, 1910).

Il est prématuré d'envisager ses rapports avec les autres représentants de la « lignée Dipetalonema 
A ce groupe se rattache Cercopithifilaria rugosicauda (Böhm et Supperer, 1953) n. comb., des Cervidés d'Europe.

4 L'espèce parasite de l'Athérure, Cercopithifilaria gabonensis n. sp.

5 L'espèce parasite des Didelphis en Amérique du Nord et du Sud, Cercopithifilaria didelphis (Esslinger et Smith, 1979) n. comb., dont les caractères morphologiques sont particulièrement évolués (atrophie de la capsule buccale, grande réduction du nombre des papilles caudales, spicules très peu cuticularisés). Cette espèce pourrait être une capture récente à partir de Cercopithifilaria holarctiques.

6 Le parasite d'Écureuil en Indes, D. laemmleri Dasgupta et coll., 1978, est peut-être aussi un Cercopithifilaria, mais la description comporte des contradictions entre figures et texte et on ne sait pas si l'œsophage est long ou court.

7 Les espèces parasites de Muridés ou d'Isoodon en Australie, Cercopithifilaria johnstoni (Mackerras, 1954) n. comb. et C. pearsoni (Spratt et Varughese, 1975) n. comb., que nous avions groupées précédemment parmi les Chenofilaria.

C - Le genre Monanema défini par un ensemble de caractères : présence d'un cou, grande cavité buccale, œsophage non divisé, papilles postcloacales étagées le long dela queue, mâle sans languettes.

Il est parasite de Sciuridés en Amérique du Nord : M. marmottae (Webster, 1967), et de Muridés en région éthiopienne : $M$. globulosa (Muller et Nelson, 1975) et $M$. nilotica El Bihari et coll., 1977.

Le genre est interprété comme dérivé d'Acanthocheilonema éthiopiens.

D - Les genres néotropicaux interprétés comme néoendémiques.

Alors que le peuplement néotropiacl avait été jugé suffisamment homogène pour être considéré comme indépendant, il apparaît, avec les données nouvelles, que plusieurs espèces, principalement parasites de Caviomorphes, sont réellement proches des Acanthocheilonema éthiopiens. Il faut donc admettre que les Filaires sont passées d'Afrique au Brésil à la fin de l'Éocène, comme cela a dû être admis précédemment pour certains Trichostrongyloides et Oxyures des Caviomorphes (Durette-Desset, 1971 ; Quentin, 1973 ; Hugot, sous presse).

Cette faune se répartit en 3 groupes:

1 Le genre Molinema Freitas et Lent, 1939, parasite de Caviomorphes ; il aurait donné naissance chez les mêmes hôtes à des formes plus évoluées, le genre Ackertia Vaz, 1934 (spicules allongés, œsophage non divisé ; papilles uniquement précloacales groupées sur la ligne médiane).

2 Le parasite de l'Hydrochère, D. (A.) hydrochoeri Yates, 1980. Cette espèce présente des"ressemblances particulièrement étroites avec Acanthocheilonema; elle s'en distingue toutefois par des caractères très spécialisés (multiplication des papilles précloacales, puissantes ailes latérales sur le corps, pas d'ailes sur la queue) et il nous semble préférable d'en faire le type d'un genre nouveau, Yatesia n. gen. : Onchocercinae à capsule buccale bien cuticularisée, œsophage très long et nettement divisé, 
petites languettes caudales, ailes latérales sur le corps interrompues après le cloaque, papilles précloacales nombreuses.

- Espèce-type : Yatesia hydrochoerus (Yates, 1980) n. comb.

L'hypothèse que nous formulons est donc que deux groupes différents de Filaires ont été importés directement d'Afrique au Brésil, d'une part le groupe Molinema, d'autre part la Filaire de l'Hydrochoerus.

3 Le genre Macdonaldius Khanna, 1933, parasite de Reptiles, qui serait une capture d'un parasite de Mammifères proche d'Acanthocheilonema.

\section{BIBLIOGRAPHIE}

Basson P. A., McCully M., De Vos V., Young E., Kruger S. : Some parasitic and other natural diseases of the African Elephant in the Kruger National Park. Onderstepoort J. Vet. Res., I97I, $38,239-254$.

Baylis H. A. : A Filariid from the African Elephant. Ann. Mag. Nat. Hist. (Ser. 9), 1923, II, 2082 II.

Baylis H. A. : The Fauna of British India, including Ceylon and Burma. Nematoda. Vol. II. (Filarioidea, Dioctophymoidea and Trichinelloidea). Taylor and Francis, Ltd, London, 1939, $274 \mathrm{p}$.

Berghe L. Van den, Gillain Gr. : Sur un genre nouveau de Filaroidea chez l'Éléphant au Congo belge. Rev. Zool. Bot. Afr., 1939, 32, 388-393.

Вонм L. K. et SUPPERER R. : Beobachtungen über eine neue Filarie (Nematoda) Wehrdikmansia rugosicauda Böhm et Supperer, 1953, aus dem subkutanen Bindegewebe des Rehes. Sitz. Osterr. Akad. Wissenx Math. naturw I, 1953, 162, 95-104.

Chabaud A. G., Bain O. : La lignée Dipetalonema. Nouvel essai de classification. Ann. Parasitol., Hum. Comp., I976, s1, 365-397.

Chabaud A. G., Landau I., Petit G. : Deux Filaires de Céphalophes au Gabon. Ann. Parasitol. Hum. Comp., 1978, s3, 385-29o.

Dasgupta S., Dasgupta B., Chatterjee A., Den C., Nandi J., Sha K., Roy R., Ghosh M., Majumbar G. : Dipetalonema laemmleri sp. nov. (Nematoda : Dipetalonematidae) from the Himalayan flying squirrel, Petaurista magnificus. Z. f. Parsit., 1978, 55, 195-198.

DuRETTE-Desset M. C. : Essai de classification des Nématodes Heligmosomes. Corrélations avec la paléographie des hôtes. Mém. Mus. Nat. Hist. Nat., $\mathrm{n}^{\text {tle }}$ sér., Ser. A, Zool, 1971, 49, I-126.

EBerhard M. L. : Dipetalonema (Cercopithifilaria) kenyensis subgen. et sp. n. (Nematoda : Filarioidea) from African Baboons, Papio anubis. J. Parasitol., 1980, 66, 551-554.

EBerHARD M. L. : Dipetalonema (Dasypafilaria) averyi subgen. et sp. n. (Nematoda : Filarioidea) from the nine-banded armadillo, Dasypus novemoinctus in Louisiana. J. Parasitol., I982, 68, 325-328.

El Bihari S., Hussein H. S., Muller R. : Monanema nilotica n. sp. (Nematoda : Onchocercidae) a cardiopulmonary parasite of the Nile rat in Sudan. J. Helm., $1977,51,317-34 \mathrm{I}$,

Esslinger J. H., Smith J. L. : Dipetalonema (Acanthocheilonema) didelphis sp. n. (Nematoda : Filarioidea) from opossums, with a redescription of D. (A.) pricei (Vaz and Pereira, 1934). J. Parasitol., 1979, 65, 928-933.

FaIN A. : Vers nouveaux de l'Okapi. Rev. Zool. Bot. Afr., 1948, 4I, 222-23o.

FAIN A., HERIN V. : Filarioses des bovidés au Ruanda-Urundi. III Etude parasitologique. Ann. Soc. Belge Med. Trop., 1955, 35, 535-554.

FaIN A. : Parasitisme intradermique par les Nématodes chez les Bovins au Ruanda. Description de deux nouvelles espèces. Ann. Soc. Belge Med. Trop., I977, 57, II 3-120.

Hoffstetter R. : Phylogénie des Mammifères : méthodes d'étude, résultats, problèmes, p. 13I9 in $2^{\mathrm{e}}$ symposium sur la Spécificité parasitaire des parasites de Vertébrés, I3-I 7 avril 198 I, Mem. Mus. Nat. Hist. Nat., ser. A, Zool., 1982, 123,326 p.

Hugot J. P. : Sur le genre Wellcomia (Oxyuridae) parasite de Rongeurs archaïques. Bull. Mus. Nat. Hist. Nat. (sous presse).

KhaliL M. B. : A revision of the Nematode Parasites of Elephants, with a description of four new species. Proc. Zool. Soc., London, 1922, I-75.

Kou C. : Studies on parasitic Nematodes of Mammals from Canton. I Some new species from Pavadoxurus minor axitus Schwarj, Paguma larvata larvata (Hamilton Smith) and Manis pentadactyla aurita Hogson. Acta Zool. Sinica, 1958, 10, 60-67.

Leiper R. T. : The entozoa of the Hippopotamus. Proc. Zool. Soc., London, 1910, 223-251. 
Meszaros F., Sugar L. : Dipetalonema rugosicauda (Böhm and Supperer, I953) nov. comb. ; occurence in roe deer in Hungary. Parasitol. Hungarica., 1976, 9, 67-71.

MonNig H. O. : Helminthological notes. $I I$ th and $I$ th Report of the Director of Veterinary Education and Research, Pretoria, I927, part I, 22I-228.

Noe G. : La Filaria grasii (Noe, I907). Ricerche lab. Anat. Norm. R. Univ. Roma, I910-I9II, Is, 235-252.

OrtLepp R. J. : On two Rictularias and a Filariid from South-african wild Carnivores. J. Helm. Suppl. Leiper, I96I, I3I-I40.

Quentrn J. C.: Affinité entre les Oxyures parasites de rongeurs Hystricidés, Erethizontidés, et Dinomydés. Intérêt paléobiogéographique. C. R. Acad. Sci., I973, 276, sér. D., 2015-20I 7.

Spratt D. M., VARUghese G. : A taxonomic revision of Filarioid Nematodes from Australian Marsupials. Austr. J. Zool., 1975, Suppl. Sér. (n 35), I-99.

YAtes J. A. : Filariae of the capybara Hydrochoerus hydrochoeris from Colombia, South America. Dissertation, 26 juin 1980 , Tulane University Dr. Philosophy, 8I p. polycopiées. 